\title{
Harnack type estimates for nonlinear elliptic systems and applications
}

\section{Inégalités de type Harnack pour les systèmes elliptiques non-linéaires et applications}

\author{
Jérôme Busca ${ }^{\mathrm{a}, *}$, Boyan Sirakov ${ }^{\mathrm{b}}$ \\ ${ }^{a}$ CNRS \& Ceremade, Université Paris Dauphine, pl. du maréchal de Lattre de Tassigny, 75775 Paris cedex 16, France \\ ${ }^{\mathrm{b}}$ UFR SEGMI, bâtiment G, Université Paris X, 200, av. de la République, 92001 Nanterre cedex, France
}

Received 3 December 2001; accepted 12 June 2003

Available online 24 October 2003

\begin{abstract}
In this paper we obtain a generalised maximum principle of Alexandrov-Bakelman-Pucci type for viscosity solutions of fully nonlinear cooperative elliptic systems. We also establish a Harnack estimate for such systems and give some applications. In particular, a Harnack type estimate for solutions of higher order equations is given.

(c) 2004 L'Association Publications de l'Institut Henri Poincaré. Published by Elsevier B.V. All rights reserved
\end{abstract}

\section{Résumé}

Dans cet article nous obtenons un principe de maximum généralisé de type Alexandrov-Bakelman-Pucci pour les solutions de viscosité de systèmes elliptiques coopératifs complètement non-linéaires. Nous établissons une inégalité de Harnack pour ce type de systèmes et présentons quelques applications. En particulier, nous obtenons une inégalité de type Harnack pour les solutions d'équations polyharmoniques.

(C) 2004 L'Association Publications de l'Institut Henri Poincaré. Published by Elsevier B.V. All rights reserved

\section{Part I. The basic estimates}

\section{Introduction}

During the last twenty years there have been many attempts to extend to weakly coupled cooperative elliptic systems of second order the theory of scalar elliptic equations in non-divergence form. This work is a contribution to this study. We establish counterparts, for cooperative fully nonlinear elliptic systems, of the fundamental Alexandrov-Bakelman-Pucci and Harnack-Krylov-Safonov estimates for scalar linear equations (see, for example, [22]).

\footnotetext{
* Corresponding author.

E-mail addresses: busca@ceremade.dauphine.fr (J. Busca), Boyan.Sirakov@ehess.fr (B. Sirakov).
} 
We study the system

$$
\left\{\begin{aligned}
F_{1}\left(D^{2} u_{1}, D u_{1}, u_{1}, \ldots, u_{n}, x\right) & =f_{1}(x) \\
F_{2}\left(D^{2} u_{2}, D u_{2}, u_{1}, \ldots, u_{n}, x\right) & =f_{2}(x) \\
\vdots & \\
F_{n}\left(D^{2} u_{n}, D u_{n}, u_{1}, \ldots, u_{n}, x\right) & =f_{n}(x)
\end{aligned}\right.
$$

in a bounded domain $\Omega \subset \mathbb{R}^{N} ; n, N \geqslant 1$. Here $F_{i}$ are uniformly elliptic fully nonlinear operators.

We obtain the following two results (see Section 3 for precise statements). First, we prove an AlexandrovBakelman-Pucci (ABP) type inequality, which has the form

$$
\sup _{x \in \Omega} \max _{1 \leqslant i \leqslant n} u_{i}(x) \leqslant \sup _{x \in \partial \Omega} \max _{1 \leqslant i \leqslant n} u_{i}(x)+C\left\|\max _{1 \leqslant i \leqslant n} f_{i}(x)\right\|_{L^{N}(\Omega)},
$$

provided $\left(u_{1}, \ldots, u_{n}\right)$ is a subsolution of (1) and (1) is coercive in an appropriate sense. Second, we obtain a Harnack inequality which states that any nonnegative solution of (1) satisfies

$$
\sup _{x \in B} \max _{1 \leqslant i \leqslant n} u_{i}(x) \leqslant \Phi\left(\inf _{x \in B} \min _{1 \leqslant i \leqslant n} u_{i}(x),\left\|\max _{1 \leqslant i \leqslant n} f_{i}(x)\right\|_{L^{N}(\Omega)}\right),
$$

where $B$ is a ball included in $\Omega$ and $\Phi(\cdot, \cdot)$ is a continuous function such that $\Phi(0,0)=0$.

For such a general system to satisfy ABP and Harnack estimates there are two unavoidable structural assumptions one is obliged to make. First, the coupling in the system appears only in the zero order terms, that is, the $i$-th equation in (1) involves only derivatives of $u_{i}$. This property is usually referred to as weak coupling. Second, the system is cooperative (quasi-monotone) in the sense that $F_{i}$ is non-decreasing in $u_{j}$, for $i \neq j$. In general, if any of these properties is not satisfied then the system does not satisfy even the maximum principle (see the counterexamples at the end of Section 3).

Harnack estimates have been essential in many areas of PDE's, such as existence and regularity of solutions of nonlinear elliptic equations, Liouville type theorems, qualitative properties of solutions. In particular, they have been the core of the theory of strong (i.e., $W_{\mathrm{loc}}^{2, N}$ ) solutions of scalar equations in non-divergence form, developed by Krylov and Safonov in the late 70's (see [30]). This theory is the counterpart of the classical De-Giorgi-NashMoser regularity theory for divergence form equations (see, for instance, [22]). A general regularity result for nonlinear equations in divergence form was obtained by Serrin in [38].

Ever since DeGiorgi's counterexample (see [17,21]) it has been known that general systems in divergence form do not enjoy the same regularity properties as scalar equations. Consequently, a great amount of work has been devoted to determining under what restrictions systems in divergence form do behave like scalar equations, as far as regularity is concerned. A basically optimal regularity result for diagonal type systems in divergence form was obtained by Hildebrandt and Widman in [26]. For a thorough account on the regularity theory for elliptic systems in divergence form we refer to Giaquinta's book [21].

On the other hand, relatively little is known about elliptic estimates for systems in non-divergence form (there have been only partial results for linear systems with regular coefficients, see the discussion after Corollary 8.1). We give here an appropriate framework in which such estimates can be obtained. Our results are complete in the sense that they reduce to those of Krylov and Safonov when $n=1$ (scalar case). This paper is the first in a program aimed at establishing a satisfactory elliptic theory for systems of type (1).

The leading idea of our work is to use the properties of viscosity solutions of partial differential equations. The viscosity solutions theory developed very rapidly during the last twenty years (we quote some of the fundamental works on the subject in Section 4). Viscosity solutions offer a number of advantages and provide a convenient framework for studying fully nonlinear equations. In addition, recent developments - the so-called $L^{N}$-viscosity solutions - permit to treat equations with discontinuous coefficients; in this setting strong solutions are a subclass of the class of $L^{N}$-viscosity solutions. 
In recent years elliptic estimates were obtained for viscosity solutions of fully nonlinear scalar equations. It turns out that viscosity solutions are an appropriate framework for studying systems too. The first to use viscosity solutions in the general setting of systems that we consider were Ishii and Koike [27], who obtained existence and uniqueness results for viscosity solutions of cooperative elliptic systems through a Perron-type argument. Our approach contains a new idea, which consists in relating system (1) to a set of scalar fully nonlinear elliptic equations and then using the Alexandrov-Bakelman-Pucci and Harnack estimates for viscosity solutions of such equations.

Although cooperative elliptic systems share many properties with scalar equations, it would definitely be wrong to think that these systems boil down to scalar equations. For example, even basic concepts in the framework of scalar equations, such as coercivity, do not admit clear (or unique) equivalents for systems. We give various conditions under which a cooperative system satisfies the maximum principle, together with counterexamples (Sections 3 and 10).

Our results are new even in the particular case of a linear system. Because of the importance of this case we have devoted to it a whole part of the article in which we restate our results in a more precise manner (Section 8). For example, we give a detailed description of the way the coupling in the system reflects into the Harnack estimate.

Further, in the linear setting we are able to give a complete answer to the coercivity issue we mentioned above. Specifically, in Section 14, we obtain a necessary and sufficient condition for the maximum principle to hold for a linear system (this question has been open for some time in the non-divergence case). This is done in terms of a properly defined first eigenvalue of the system. Actually, it was not known before whether a general cooperative system admits a first eigenvalue with properties similar to those of the first eigenvalue of a scalar operator (except for a partial and somewhat different result by Hess).

An application of our results are ABP and Harnack estimates for higher order equations such as

$$
\Delta^{n} u=f .
$$

Estimates for polyharmonic functions $(f \equiv 0)$ are a very classical problem. Even though their study dates back to the nineteenth century, such estimates were obtained much later, and they depend on the polyharmonicity of the function. A Harnack estimate was available neither for more general higher order equations, nor for equations with a right-hand side. See Section 15 for a discussion and results.

\section{Examples}

We give here a selection of problems, taken from different fields, which lead to weakly coupled cooperative elliptic systems of type (1).

Switched diffusion processes (probability theory). Let $\lambda_{t} \in\{1, \ldots, n\}$ be a discrete valued Markov process and let $X_{t}$ be a diffusion process such that

$$
\mathrm{d} X_{t}=b^{\lambda_{t}} \mathrm{~d} t+\sigma^{\lambda_{t}} \mathrm{~d} W_{t},
$$

where $W_{t}$ is a standard $n$-dimensional Wiener process, independent of $\lambda_{t}$. Suppose $p_{i j} \geqslant 0$ is the probability of transition from state $i$ to state $j$ of $\lambda_{t}$. Then

$$
u_{i}(x)=\mathbb{E}^{(x, i)}\left[\phi_{\lambda_{\tau(\Omega)}}\left(X_{\tau(\Omega)}\right)\right], \quad i=1, \ldots, n,
$$

is a solution of the linear system

$$
\begin{cases}L_{i} u_{i}+\sum_{j \neq i} p_{i j} u_{j}=0 & \text { in } \Omega, \\ u_{i}=\phi_{i} & \text { on } \partial \Omega, \quad i=1, \ldots, n,\end{cases}
$$

where

$$
L_{i} w=\frac{1}{2} \operatorname{tr}\left(\sigma^{i}\left(\sigma^{i}\right)^{t} D^{2} w\right)+b^{i} \cdot D w
$$


and $\tau(\Omega)$ is the first exit time of $X_{t}$ from $\Omega$. See [11] for a more detailed description of the problem.

Jumping volatility models (mathematical finance). In this well-known extension of the Black-Scholes pricing model the underlying asset follows the stochastic differential equation

$$
\mathrm{d} S_{t}=r S_{t} \mathrm{~d} t+\sigma^{\lambda_{t}} S_{t} \mathrm{~d} W_{t},
$$

under the risk-neutral probability. Here $r$ denotes the risk-free rate, $\sigma$ is the volatility of the asset ( $\sigma$ can take $n$ different values) and $\lambda_{t} \in\{1, \ldots, n\}$ is a discrete-valued Markov process, independent of the standard onedimensional brownian motion $W_{t}$. If we denote the price of an European call option with maturity $T$ and strike $K$ by $C\left(t, S_{t}, \lambda_{t}\right)$, then the function

$$
C_{i}(t, S)=C(t, S, i), \quad(t, S) \in[0, T] \times \mathbb{R}^{+},
$$

is shown to satisfy the system

$$
\left\{\begin{array}{l}
\frac{\partial C_{i}}{\partial t}+\frac{\left(\sigma^{i}\right)^{2}}{2} S^{2} \frac{\partial^{2} C_{i}}{\partial S^{2}}+r S \frac{\partial C_{i}}{\partial S}-r C_{i}+\frac{1}{\rho} \sum_{j \neq i} p_{i j}\left(C_{j}-C_{i}\right)=0, \\
C_{i}(T, S)=(S-K)^{+},
\end{array}\right.
$$

where $p_{i j}$ is as above and $\rho$ is the characteristic time of $\lambda_{t}$. For details on this model, see [4,5,31].

Remark. Strictly speaking, the above system falls out of the scope of our work, since it is parabolic (or degenerate elliptic). We consider it as a motivation for an extension of our work to parabolic systems. We do not doubt such an extension is possible.

Mathematical biology. A number of models in population dynamics lead to elliptic and parabolic systems which can be transformed into cooperative systems of type (1). A simple example is the system

$$
\left\{\begin{array}{l}
\Delta u+u(a-b u-c v)=0, \\
\Delta v+v(d-e u-f v)=0
\end{array} \text { in } \Omega .\right.
$$

We refer to the abundant literature on this topic, for example, [34].

Switching games (stochastic games and control theory). A typical example is the system

$$
\begin{cases}\max \left\{L_{i} u_{i}-f_{i},-u_{i}+M_{i} u\right\}=0 & \text { in } \Omega, i=1, \ldots, n, \\ u_{i}=0 & \text { on } \partial \Omega, i=1, \ldots, n,\end{cases}
$$

where $L_{i}$ are uniformly elliptic linear operators, $u=\left(u_{1}, \ldots, u_{n}\right)$, and

$$
M_{i} u(x)=\max _{j \neq i}\left\{-k_{i j}+u_{j}(x)\right\}, \quad \text { for some } k_{i j} \in \mathbb{R} .
$$

This problem arises when considering a system whose state processes are of Ito type and who can be switched into $n$ different regimes. The problem is then to choose an appropriate switching so as to minimise the resulting cost. See [32] for details on this problem.

Other problems from stochastic games theory lead to the more general system

$$
\min \left\{\max \left\{G_{i}\left(D^{2} u_{i}, D u_{i}, u_{i}, x\right),-u_{i}+M_{i}(u, x)\right\},-u_{i}+N_{i}(u, x)\right\}=0,
$$

$i=1, \ldots, n$, where

$$
M_{i}(u, x)=\max _{j \neq i}\left\{u_{j}+g_{i j}(x)\right\}, \quad N_{i}(u, x)=\min _{j \neq i}\left\{u_{j}+h_{i j}(x)\right\},
$$

with $g_{i j}, h_{i j} \in C(\Omega)$. See [27] and the references therein. 


\section{Main results}

We study the system

$$
\left\{\begin{array}{l}
F_{i}\left(D^{2} u_{i}, D u_{i}, u_{1}, \ldots, u_{n}, x\right)=f_{i}(x) \\
i=1, \ldots, n
\end{array}\right.
$$

defined in a bounded domain $\Omega \subset \mathbb{R}^{N} ; n, N \geqslant 1$.

The elliptic operators $F_{1}, \ldots, F_{n}$, defined on $\mathcal{S}_{N}(\mathbb{R}) \times \mathbb{R}^{N} \times \mathbb{R}^{n} \times \Omega\left(\mathcal{S}_{N}(\mathbb{R})\right.$ denotes the space of real symmetric $N \times N$ matrices) are supposed to satisfy the following set of assumptions. First, we assume that there exist constants $\alpha_{0} \in(0,1), \gamma \geqslant 0$ and measurable functions $c_{i}, g_{i}: \mathbb{R}^{n} \times \Omega \rightarrow \mathbb{R}, i=1, \ldots, n$, such that

(H0) $c_{i}(u, x), g_{i}(u, x)$ are globally Lipschitz continuous in $u \in \mathbb{R}^{n}$, uniformly in $x \in \Omega \backslash \mathcal{N}$ for some Lebesgue null set $\mathcal{N} \subset \Omega$, with Lipschitz constant $v$ (in the sense that the $l_{1}$-norms of $\nabla_{u} c_{i}$ and $\nabla_{u} g_{i}$ are bounded by $v)$;

(H1) $F_{i}(M, p, u, x) \leqslant \sup _{A \in \mathcal{A}} \operatorname{tr}(A M)+\gamma|p|+c_{i}(u, x), i=1, \ldots, n$;

(H2) $F_{i}(M, p, u, x) \geqslant \inf _{A \in \mathcal{A}} \operatorname{tr}(A M)-\gamma|p|+g_{i}(u, x), i=1, \ldots, n$,

for all $(M, p, u) \in \mathcal{S}_{N}(\mathbb{R}) \times \mathbb{R}^{N} \times \mathbb{R}^{n}$ and a.e. $x \in \Omega$, where $\mathcal{A}$ denotes the set of all symmetric matrices whose eigenvalues lie in the interval $\left[\alpha_{0}, \alpha_{0}^{-1}\right]$. Elliptic estimates have been established for scalar equations which satisfy the above hypotheses (see Section 4). Without restricting the generality we can suppose that $c_{i}(0, x)=g_{i}(0, x)=0$, for a.e. $x \in \Omega$ and all $i$.

We assume that system (1) is cooperative (or quasi-monotone), in the following sense: for any $u, v \in \mathbb{R}^{n}$ such that $u \geqslant v$ component-wise and any $j \in\{1, \ldots, n\}$ for which $u_{j}=v_{j}$, we have

(H3) $c_{j}(u, x) \geqslant c_{j}(v, x)$ and $g_{j}(u, x) \geqslant g_{j}(v, x)$ for a.e. $x \in \Omega$.

We study $L^{N}$-viscosity solutions of (1), that is, vector functions

$$
u=\left(u_{1}, \ldots, u_{n}\right) \in C\left(\Omega, \mathbb{R}^{n}\right)
$$

satisfying (1) in a sense that we make precise in Section 4 (see Definition 4.2). In particular, any strong solution of (1) (that is, any $u \in W_{\text {loc }}^{2, N}\left(\Omega, \mathbb{R}^{n}\right)$, satisfying (1) pointwise a.e. in $\Omega$ ) is a $L^{N}$-viscosity solution; see Section 4 , Proposition 4.2. We make the convention that, throughout the paper, all differential equations and inequations are assumed to hold in the $\left(L^{N}\right)$-viscosity sense, unless otherwise stated. Besides, all relations between vectors are understood to hold component-wise.

We use the following notations

$$
\begin{aligned}
& v \vee w(x)=\max \{v(x), w(x)\}, \quad v \wedge w(x)=\min \{v(x), w(x)\}, \\
& v^{+}(x)=\max \{v(x), 0\}, \quad v^{-}(x)=\max \{-v(x), 0\},
\end{aligned}
$$

for any two functions $v$ and $w$.

We suppose that the right-hand side of (1) satisfies

(H4) $f_{i} \in L^{N}(\Omega), i=1, \ldots, n$,

and set $f=f_{1} \vee \cdots \vee f_{n}$. 
Our first result is an Alexandrov-Bakelman-Pucci (ABP) type estimate. To our knowledge, this is the first estimate of this kind for systems of type (1)

Theorem 3.1 (ABP estimate). We assume (H0), (H1), (H3) and (H4). Let $u \in C\left(\bar{\Omega}, \mathbb{R}^{n}\right)$ satisfy

$$
\left\{\begin{array}{l}
F_{i}\left(D^{2} u_{i}, D u_{i}, u_{1}, \ldots, u_{n}, x\right) \geqslant-f_{i}(x) \text { in } \Omega, \\
i=1, \ldots, n
\end{array}\right.
$$

In addition, we assume that either

(H5) for all $i=1, \ldots, n$

$$
\sum_{j=1}^{n} \frac{\partial c_{i}}{\partial u_{j}}(u, x) \leqslant 0 \quad \text { a.e. in } \mathbb{R}^{n} \times \Omega
$$

or

(H6) if we set

$$
\bar{m}_{i j}=\operatorname{supess}_{(u, x) \in \mathbb{R}^{n} \times \Omega} \frac{\partial c_{i}}{\partial u_{j}}(u, x)
$$

$\left(\bar{m}_{i j} \leqslant \nu<\infty\right)$ then the matrix $\bar{M}=\left(\bar{m}_{i j}\right)_{i, j=1}^{n}$ is negative semi-definite, that is, $(\bar{M} \xi, \xi) \leqslant 0$ for all $\xi \in \mathbb{R}^{n}$.

Then the following ABP inequality holds

$$
\sup _{\Omega}\left(u_{1} \vee \cdots \vee u_{n}\right) \leqslant C\left(\sup _{\partial \Omega}\left(u_{1}^{+} \vee \cdots \vee u_{n}^{+}\right)+\left\|f^{+}\right\|_{L^{N}(\Omega)}\right) .
$$

The constant $C$ depends only on $N, \alpha_{0}, \gamma, v$, and $\operatorname{diam} \Omega$.

Under (H5) we can weaken (H0), namely we can suppose that $c_{i}$ are only locally Lipschitz in $u$. Furthermore, under (H5) the following stronger conclusion holds true

$$
\sup _{\Omega} u_{1} \vee \cdots \vee u_{n} \leqslant \sup _{\partial \Omega} u_{1}^{+} \vee \cdots \vee u_{n}^{+}+C_{\mathrm{ABP}}\left\|f^{+}\right\|_{L^{N}(\Omega)},
$$

where $C_{\mathrm{ABP}}$ depends only on $N, \alpha_{0}, \gamma$, and $\operatorname{diam} \Omega$.

Theorem 3.1bis (ABP estimate). We assume $(\mathrm{H} 0),(\mathrm{H} 2),(\mathrm{H} 3),(\mathrm{H} 4)$ and either $(\mathrm{H} 5)$ or $(\mathrm{H} 6)$, with $c_{i}$ replaced by $g_{i}$. Let u satisfy

$$
\left\{\begin{array}{l}
F_{i}\left(D^{2} u_{i}, D u_{i}, u_{1}, \ldots, u_{n}, x\right) \leqslant f_{i}(x) \text { in } \Omega \\
i=1, \ldots, n
\end{array}\right.
$$

Then

$$
-\inf _{\Omega}\left(u_{1} \wedge \cdots \wedge u_{n}\right) \leqslant C\left(\sup _{\partial \Omega}\left(u_{1}^{-} \vee \cdots \vee u_{n}^{-}\right)+\left\|f^{+}\right\|_{L^{N}(\Omega)}\right),
$$

where $C$ depends only on $N, \alpha_{0}, \gamma, \nu$, and $\operatorname{diam} \Omega$.

Under (H5) we can suppose that $g_{i}$ are only locally Lipschitz in $u$. Furthermore, under (H5) the following stronger conclusion holds true

$$
-\inf _{\Omega}\left(u_{1} \wedge \cdots \wedge u_{n}\right) \leqslant \sup _{\partial \Omega}\left(u_{1}^{-} \vee \cdots \vee u_{n}^{-}\right)+C_{\mathrm{ABP}}\left\|f^{+}\right\|_{L^{N}(\Omega)},
$$

where $C_{\mathrm{ABP}}$ depends only on $N, \alpha_{0}, \gamma$, and $\operatorname{diam} \Omega$. 
Remark 1. In the particular case $f \equiv 0$ Theorems 3.1 and 3.1 bis yield a maximum principle for the nonlinear system (1).

Remark 2. Neither of hypotheses (H5) and (H6) contains the other, as the following example shows. Take the following two couples of functions (with $n=2, u=\left(u_{1}, u_{2}\right)$ )

$$
\left\{\begin{array} { l } 
{ c _ { 1 } ^ { ( 1 ) } ( u , x ) = a ( x ) ^ { - 1 } ( - u _ { 1 } + u _ { 2 } ) , } \\
{ c _ { 2 } ^ { ( 1 ) } ( u , x ) = a ( x ) ( u _ { 1 } - u _ { 2 } ) , }
\end{array} \quad \left\{\begin{array}{l}
c_{1}^{(2)}(u, x)=-2 u_{1}+3 \arctan u_{2}, \\
c_{2}^{(2)}(u, x)=\arctan u_{1}-2 u_{2},
\end{array}\right.\right.
$$

where $a(x)$ is a continuous function from $\Omega$ onto $\left[\frac{1}{2}, 2\right]$. Then the first couple satisfies (H5) but not (H6), while the second couple satisfies (H6) but not (H5).

Note that both (H5) and (H6) are hypotheses on the matrix

$$
\mathcal{C}(u, x)=\left(\frac{\partial c_{i}}{\partial u_{j}}(u, x)\right)_{i, j=1}^{n} .
$$

A natural way to unify and extend these two hypotheses would be to suppose that the matrix $\mathcal{C}(u, x)$ itself is negative semi-definite, for almost every $(u, x)$. Indeed, one can see, under (H3), that this condition is implied by either of (H5) and (H6), see Lemma 10.1. However, it turns out that the ABP inequality (and even the maximum principle) fails if we make this assumption only. See Section 10 for a counterexample.

Further, we establish a Harnack inequality for non-negative solutions of system (1).

The form of the Harnack inequality depends very much on the way the system relates the functions $u_{1}, \ldots, u_{n}$ to each other. Here, for simplicity, we shall suppose that system (1) links all functions $u_{1}, \ldots, u_{n}$ in a strong sense, or, more precisely, that system (1) is fully coupled. We give a suitable nonlinear meaning to this notion. Note that the assumption of full coupling can be removed and the result can be made much more precise - nevertheless, to avoid heavy notations here, we state these more general results in the simplified framework of linear systems (see Section 8).

Fix indices $k, l \in\{1, \ldots, n\}$ such that $k \neq l$ and let $\omega_{k l}$ be a non-null measurable subset of $\Omega$. We define the function

$$
\varphi_{k l}(t)=\inf _{x \in \omega_{k l}} \operatorname{ess}_{k}\left(t e_{l}, x\right), \quad \text { for } t \geqslant 0,
$$

where $e_{l} \in \mathbb{R}^{n}$ is the vector with $l$-th coordinate equal to one and all other coordinates equal to zero. For simplicity of notation we shall not write explicitly the dependence of $\varphi_{k l}$ on $\omega_{k l}$. One can check that (H0) implies that $\varphi_{k l}$ is globally Lipschitz continuous on $[0,+\infty)$. Note that $\varphi_{k l}$ is non-decreasing, because of $(\mathrm{H} 3)$, and $\varphi_{k l}(0)=0$.

The following definition provides a nonlinear version of the commonly used notion of full coupling.

Definition 3.1. We call system (1) fully coupled in $\Omega$, provided for any non-empty sets $I, J \subset\{1, \ldots, n\}$ such that $I \cap J=\emptyset$ and $I \cup J=\{1, \ldots, n\}$, there exist $i_{0} \in I$ and $j_{0} \in J$ for which one can find a set $\omega_{i_{0} j_{0}} \subset \Omega$ with positive Lebesgue measure such that $\varphi_{i_{0} j_{0}}(t)$ does not vanish for $t \neq 0$. Under (H3) this means that

$$
\varphi_{i_{0} j_{0}}(t)>0 \text { for all } t>0 \text {. }
$$

In some sense, a system is fully coupled if it cannot be split into two subsystems, one of which does not depend on the other. Note that any scalar equation is a fully coupled system.

Theorem 3.2 (Harnack inequality). Suppose (H0) through (H4) hold and let $u \geqslant 0$ be a solution of (1) in a ball $B_{3 R} \subset \Omega$. Suppose, in addition, that system (1) is fully coupled in $B_{R}$. Then there exists a non-negative continuous function $\Phi: \mathbb{R}_{+}^{2} \rightarrow \mathbb{R}_{+}$, with $\Phi(0,0)=0$, depending only on $\left\{R^{2} \varphi_{i j}\right\}, N, n, \alpha_{0}, \gamma R, v R^{2}$, such that

$$
\sup _{B_{R}} u_{1} \vee \cdots \vee u_{n} \leqslant \Phi\left(\inf _{B_{R}} u_{1} \wedge \cdots \wedge u_{n}, R\|f\|_{L^{N}\left(B_{3 R}\right)}\right) .
$$


Remark 1. An explicit expression of $\Phi$ is given in the proof of Theorem 3.2. Roughly speaking, $\Phi(t, 0)$ is a composition of the inverse functions of the functions $\varphi_{k l}(t)$. For example, if $\varphi_{k l}(t) \geqslant c t^{\alpha}$ for some $\alpha>0$ and all indices $k \neq l$, then $\Phi(t, 0) \leqslant C t^{\alpha^{-n(n-1)}}$.

Remark 2. In the particular case of a linear system $\Phi(t, s)=C_{1} t+C_{2} s$ (see Section 8, Theorem 8.2 and Corollary 8.1).

In the course of the proof of the Harnack estimate we obtain two estimates of independent interest, for subsolutions and supersolutions of system (1). These have well-known counterparts in the scalar case too.

We set, for any $p>0$,

$$
|u|_{p, R}=\left(\frac{1}{\operatorname{meas}\left(B_{R}\right)} \int_{B_{R}}|u|^{p}\right)^{1 / p} .
$$

Proposition 3.1 (local maximum principle). Suppose (H0), (H1), (H3), and (H4) hold. Let $u \in C\left(B_{3 R}, \mathbb{R}^{n}\right)$ be a solution of

$$
\left\{\begin{array}{l}
F_{i}\left(D^{2} u_{i}, D u_{i}, u_{1}, \ldots, u_{n}, x\right) \geqslant-f_{i}(x), \\
i=1, \ldots, n
\end{array}\right.
$$

in $B_{3 R}$. Then for all $p>0$ we have

$$
\sup _{B_{R}} u_{1} \vee \cdots \vee u_{n} \leqslant C\left(\left|u_{1}^{+} \vee \cdots \vee u_{n}^{+}\right|_{p, 2 R}+R\left\|f^{+}\right\|_{L^{N}\left(B_{2 R}\right)}\right),
$$

where $C=C\left(N, \alpha_{0}, p, \gamma R, \nu R^{2}\right)$.

Proposition 3.2 (weak Harnack inequality). Suppose (H0), (H2), (H3), and (H4) hold. Assume (1) is fully coupled and let $u \in C\left(B_{3 R}, \mathbb{R}^{n}\right)$ be a non-negative solution of

$$
\left\{\begin{array}{l}
F_{i}\left(D^{2} u_{i}, D u_{i}, u_{1}, \ldots, u_{n}, x\right) \leqslant f_{i}(x), \\
i=1, \ldots, n
\end{array}\right.
$$

in $B_{3 R}$. Then there exists a number $p=p\left(N, n, \alpha_{0}, \gamma R, v R^{2}\right)>0$ such that

$$
\left|u_{1} \vee \cdots \vee u_{n}\right|_{p, 2 R} \leqslant \Phi\left(\inf _{B_{R}} u_{1} \wedge \cdots \wedge u_{n}, R\|f\|_{L^{N}\left(B_{3 R}\right)}\right),
$$

where $\Phi$ is as in Theorem 3.2 .

Counterexample 1. All our results fail for general non-cooperative systems. Simple examples are provided by the systems

$$
\left\{\begin{array} { l } 
{ \Delta u - v = 0 , } \\
{ \Delta v = 0 , }
\end{array} \quad \left\{\begin{array}{l}
\Delta u-v=0, \\
\Delta v-u \leqslant 0,
\end{array} \text { in } B_{1} \subset \mathbb{R}^{N} .\right.\right.
$$

The first system satisfies all hypotheses of Theorem 3.1 except for (H3). By taking $u=1-|x|^{2}, v=-2 N$, we see that Theorem 3.1 (with $f \equiv 0$ ) is false for this system since $u=0, v \leqslant 0$ on $\partial B_{1}$ but $u \nless 0$ in $B_{1}$. A counterexample for the weak Harnack inequality (Proposition 3.2) is obtained by setting $u=|x|^{2}, v=2 N$ in the second system, since $\inf _{B_{1}} u \wedge v=0$ but $u, v \not \equiv 0$.

Counterexample 2. There is no hope to obtain maximum principles for general systems in non-divergence form with coupling in the first-order terms. For example, consider the system of inequalities

$$
\left\{\begin{array}{l}
\Delta u+v_{x}-\alpha u \leqslant 0, \\
\Delta v+u_{x}-\alpha v \leqslant 0
\end{array} \text { in } \Omega=(-1,1) \subset \mathbb{R},\right.
$$


where $\alpha>1$ is arbitrary. The functions

$$
u(x)=\left\{\begin{array}{ll}
0 & \text { in }(-1,0) \\
\frac{1}{4 \alpha} x(x-1) & \text { in }(0,1),
\end{array} \quad v(x)= \begin{cases}2 & \text { in }(-1,0), \\
2-x & \text { in }(0,1),\end{cases}\right.
$$

satisfy (14) and $u=0, v \geqslant 0$ on $\partial \Omega$. However $u \neq 0$ in $\Omega$. Note that the elliptic operators $F_{i}$ in (14) can be arbitrarily coercive in $u_{i}$, when $\alpha \gg 1$, yet this does not help.

Counterexample 3. This example shows that even systems of equalities do not necessarily satisfy the maximum principle if they are coupled in the first order terms.

The functions

$$
u(x, y)=x^{2}+y^{2}-1, \quad v(x, y)=-\frac{1}{3} x^{3}-4 y+5
$$

solve the system

$$
\left\{\begin{array}{l}
\Delta u+v_{y}=0, \\
\Delta v+u_{x}=0
\end{array} \text { in } B_{1} \subset \mathbb{R}^{2},\right.
$$

and $u=0, v>0$ on $\partial B_{1}$. However $u \ngtr 0$ in $B_{1}$.

Sections 4-7 are devoted to the proofs of Theorems 3.1 and 3.2.

\section{Definition and properties of viscosity solutions}

In this section we describe the class of viscosity solutions to which our results apply. The concept of viscosity solution has found many applications in PDE's (see for example the fundamental work [12]).

Basic tools in the proof of our results are the ABP estimate and the Harnack inequality for viscosity solutions of fully nonlinear scalar elliptic equations, obtained by Wang in [41], Caffarelli, Crandall, Kocan and Swiech in [9]. We shall state, for the reader's convenience, the results from these papers that we need. We refer to the book by Caffarelli and Cabre [8] for results on $C$-viscosity solutions of fully nonlinear elliptic equations.

Consider a measurable function $G: \mathcal{S}_{N}(\mathbb{R}) \times \mathbb{R}^{N} \times \mathbb{R} \times \Omega \rightarrow \mathbb{R}$, such that $G(\cdot, \cdot, \cdot, x)$ is locally uniformly continuous, uniformly for $x \in \Omega \backslash \mathcal{N}$, where $\mathcal{N}$ is a Lebesgue null set. Take a measurable function $f$ and a continuous function $w$, defined in $\Omega$. We consider the scalar equation

$$
G\left(D^{2} w, D w, w, x\right)=f \quad \text { in } \Omega,
$$

provided $G$ is uniformly elliptic, that is, there exists $\beta_{0}>0$ such that

$$
\beta_{0}\left|M^{\prime}\right| \leqslant G\left(M+M^{\prime}, P, w, x\right)-G(M, P, w, x) \leqslant \beta_{0}^{-1}\left|M^{\prime}\right|,
$$

for any matrix $M \in \mathcal{S}_{N}(\mathbb{R})$, any positive definite matrix $M^{\prime} \in \mathcal{S}_{N}(\mathbb{R})$, any $P \in \mathbb{R}^{N}, w \in \mathbb{R}$ and a.e. $x \in \Omega$. We denote by $A^{\mathrm{T}}$ the transposed matrix of $A$, and set $|A|=\sqrt{\operatorname{tr}\left(A^{\mathrm{T}} A\right)}$, for any $A \in \mathcal{M}_{N}(\mathbb{R})$.

We recall the definition of a $L^{N}$-viscosity solution of a scalar equation.

Definition 4.1 (scalar equations; [41,9]). We say that the function $w \in C(\Omega)$ is a $\left(L^{N}\right)$-viscosity subsolution (supersolution) of (15), provided for any $\varepsilon>0$, any open subset $\mathcal{O} \subset \Omega$, and any $\varphi \in W^{2, N}(\mathcal{O}$ ) (we call $\varphi$ a test function), such that

$$
\begin{aligned}
& G\left(D^{2} \varphi(x), D \varphi(x), w(x), x\right) \leqslant f(x)-\varepsilon \\
& \left(G\left(D^{2} \varphi(x), D \varphi(x), w(x), x\right) \geqslant f(x)+\varepsilon\right) \quad \text { a.e. in } \mathcal{O},
\end{aligned}
$$

the function $w-\varphi$ cannot achieve a local maximum (minimum) equal to zero in $\mathcal{O}$. In this case we say that the function $w$ satisfies the inequation 


$$
G\left(D^{2} w, D w, w, x\right) \geqslant(\leqslant) f
$$

in the $\left(L^{N}\right)$-viscosity sense in $\Omega$.

We say that $w$ is a solution of (15) if $w$ is at the same time a subsolution and a supersolution of (15).

Remark. This definition is equivalent to Definition 2.1 in [9], setting $p=N$ there. It is easy to see, under our hypotheses on $G$, that the class of viscosity solutions the above definition introduces is included in the class considered by Wang in [41].

Next, we recall the definition of the Pucci extremal operators

$$
\mathcal{M}^{+}(M)=\alpha_{0}^{-1} \sum_{e_{i}>0} e_{i}+\alpha_{0} \sum_{e_{i}<0} e_{i}, \quad \mathcal{M}^{-}(M)=\alpha_{0} \sum_{e_{i}>0} e_{i}+\alpha_{0}^{-1} \sum_{e_{i}<0} e_{i},
$$

for $M \in \mathcal{S}_{N}(\mathbb{R})$, where $e_{1}, \ldots, e_{N}$ denote the eigenvalues of $M$. Then (see [8])

$$
\mathcal{M}^{+}(M)=\sup _{A \in \mathcal{A}} \operatorname{tr}(A M), \quad \mathcal{M}^{-}(M)=\inf _{A \in \mathcal{A}} \operatorname{tr}(A M),
$$

where $\mathcal{A}$ denotes the set of all symmetric matrices whose eigenvalues lie in the interval $\left[\alpha_{0}, \alpha_{0}^{-1}\right]$. To relate our notations to those of [9] one has to note that $\mathcal{M}^{+}=-\mathcal{P}^{-}$and $\mathcal{M}^{-}=-\mathcal{P}^{+}$, with $\mathcal{P}^{+}, \mathcal{P}^{-}$defined in [9]. It is not difficult to check that (see [8])

$$
-\mathcal{M}^{+}(-M)=\mathcal{M}^{-}(M), \quad \mathcal{M}^{+}(\eta M)=\eta \mathcal{M}^{+}(M)
$$

and

$$
\begin{aligned}
& \mathcal{M}^{+}(M)+\mathcal{M}^{-}(N) \leqslant \mathcal{M}^{+}(M+N) \leqslant \mathcal{M}^{+}(M)+\mathcal{M}^{+}(N), \\
& \mathcal{M}^{-}(M)+\mathcal{M}^{-}(N) \leqslant \mathcal{M}^{-}(M+N) \leqslant \mathcal{M}^{+}(M)+\mathcal{M}^{-}(N),
\end{aligned}
$$

for every two symmetric matrices $M, N$, and every $\eta \geqslant 0$.

We define the extremal operators

$$
\begin{aligned}
& \mathcal{L}^{+}\left(D^{2} w, D w\right)=\mathcal{M}^{+}\left(D^{2} w\right)+\gamma|D w|, \\
& \mathcal{L}^{-}\left(D^{2} w, D w\right)=\mathcal{M}^{-}\left(D^{2} u\right)-\gamma|D w|
\end{aligned}
$$

$\left(\gamma\right.$ is defined in $(\mathrm{H} 1)$ and $(\mathrm{H} 2),|\cdot|$ denotes the Euclidean norm in $\left.\mathbb{R}^{N}\right)$. Note that

$$
\mathcal{L}^{+}\left(D^{2} w, D w\right)=-\mathcal{L}^{-}\left(-D^{2} w,-D w\right) .
$$

Definition 4.2 (systems). We call the vector $u \in C\left(\Omega, \mathbb{R}^{n}\right)$ a subsolution of (1) provided the equation

$$
\mathcal{L}^{+}\left(D^{2} u_{i}, D u_{i}\right) \geqslant-c_{i}(u, x)+f_{i}(x)
$$

is satisfied in the viscosity sense for each $i \in\{1, \ldots, n\}$, in terms of Definition 4.1. Equivalently, we say that $u$ satisfies the system

$$
F_{i}\left(D^{2} u_{i}, D u_{i}, u, x\right) \geqslant f_{i}(x), \quad i=1, \ldots, n .
$$

Respectively, $u \in C\left(\Omega, \mathbb{R}^{n}\right)$ is called a supersolution of (1) provided the equation

$$
\mathcal{L}^{-}\left(D^{2} u_{i}, D u_{i}\right) \leqslant-g_{i}(u, x)+f_{i}(x)
$$

is satisfied in the viscosity sense for each $i \in\{1, \ldots, n\}$, in terms of Definition 4.1. Equivalently, we say that $u$ satisfies the system

$$
F_{i}\left(D^{2} u_{i}, D u_{i}, u, x\right) \leqslant f_{i}(x), \quad i=1, \ldots, n .
$$


A solution of (1) is a vector $u \in C\left(\Omega, \mathbb{R}^{n}\right)$ which is both a subsolution and a supersolution of (1).

The rest of this section contains a list of results on viscosity solutions of scalar equations. We shall need these in the sequel.

Proposition 4.1. The maximum of two viscosity subsolutions of a scalar equation is a viscosity subsolution. The minimum of two viscosity supersolutions of a scalar equation is a viscosity supersolution.

Proof. This is very well-known and obvious, from Definition 4.1.

Proposition 4.2. Let $f \in L^{N}(\Omega)$ and let $\mathcal{L}$ be a scalar extremal operator, as in (19). Suppose that $w \in W_{\mathrm{loc}}^{2, N}(\Omega)$ is such that $\mathcal{L}\left(D^{2} w, D w\right) \geqslant(\leqslant) f$ a.e. in $\Omega$. Then $\mathcal{L}\left(D^{2} w, D w\right) \geqslant(\leqslant) f$ in the viscosity sense. Conversely, if $w \in W_{\text {loc }}^{2, N}(\Omega)$ satisfies $\mathcal{L}\left(D^{2} w, D w\right) \geqslant(\leqslant) f$ in the viscosity sense in $\Omega$ then $\mathcal{L}\left(D^{2} w, D w\right) \geqslant(\leqslant) f$ a.e. in $\Omega$.

Proof. This follows from Lemma 2.5 and Corollary 3.7 in [9].

The following lemma contains several easy, but important properties of $\mathcal{L}^{+}$and $\mathcal{L}^{-}$.

Lemma 4.1. (a) Let $f \in L^{N}(\Omega)$. Suppose $L_{k}, k=1, \ldots, n$, are linear uniformly elliptic second-order operators without zero-order terms, with ellipticity constant $\alpha_{0}$ and all their coefficients bounded by $\gamma$. Then $L_{k} w \leqslant f$, for some $k$, (resp. $\geqslant)$ implies

$$
\mathcal{L}^{-}\left(D^{2} w, D w\right) \leqslant f \quad\left(\text { resp. } \mathcal{L}^{+}\left(D^{2} w, D w\right) \geqslant f\right) .
$$

Conversely, $\mathcal{L}^{+}\left(D^{2} w, D w\right) \leqslant f$ implies $L_{k} w \leqslant f$, and $\mathcal{L}^{-}\left(D^{2} w, D w\right) \geqslant f$ implies $L_{k} w \geqslant f$, for all $k=1, \ldots, n$. This is the reason for which $\mathcal{L}^{+}$and $\mathcal{L}^{-}$are called extremal.

(b) Let $w \in W_{\text {loc }}^{2, N}(\Omega)$. There exist scalar linear uniformly elliptic second order operators $L_{0}^{+}, L_{0}^{-}$(depending on $w)$ with bounded measurable coefficients, such that

$$
\mathcal{L}^{+}\left(D^{2} w, D w\right)=L_{0}^{+} w, \quad \mathcal{L}^{-}\left(D^{2} w, D w\right)=L_{0}^{-} w .
$$

Furthermore, $\alpha_{0}$ is an ellipticity constant for the operators $L_{0}^{+}, L_{0}^{-}$, and $\gamma$ is an upper bound for the $L^{\infty}$-norms of their first order coefficients.

Proof. Part (a) is a direct consequence of (16) and Definition 4.1. Part (b) follows from the fact that the supremum and the infimum in (16) are attained (since $\mathcal{A}$ is compact). For instance, we take

$$
L_{0}^{+} w(x)=\operatorname{tr}\left(A_{0}^{+}(x) D^{2} w(x)\right)+\vec{b}(x) D w(x),
$$

where $x \rightarrow A_{0}^{+}(x)$ is a measurable selection of elements of $\mathcal{A}$ at which

$$
\sup _{A \in \mathcal{A}} \operatorname{tr}\left(A D^{2} w(x)\right)
$$

is attained, and

$$
\vec{b}(x)= \begin{cases}\gamma \frac{D w(x)}{|D w(x)|}, & \text { if } D w(x) \neq 0, \\ 0, & \text { if } D w(x)=0 .\end{cases}
$$

We shall use the following Alexandrov-Bakelman-Pucci inequality for fully nonlinear scalar equations.

Proposition 4.3. Let $w \in C(\bar{\Omega})$ and $f \in L^{N}(\Omega)$ satisfy the scalar inequality

$$
-\mathcal{L}^{+}\left(D^{2} w, D w\right) \leqslant f \quad \text { in } \Omega \cap\{w>0\} .
$$


Then

$$
\sup _{\Omega} w \leqslant \sup _{\partial \Omega} w^{+}+C_{*}\left\|f^{+}\right\|_{L^{N}\left(\Gamma^{+}\right)} .
$$

Here $\Gamma^{+}$denotes the upper contact set of $w$, defined by

$$
\Gamma^{+}=\{x \in \Omega \mid w(x)=\bar{w}(x)\},
$$

where $\bar{w}$ is the concave envelope of $w$, i.e.

$$
\bar{w}=\inf \{\widetilde{w} \mid \widetilde{w} \geqslant w \text { and } \widetilde{w} \text { is concave }\},
$$

and the constant $C_{*}$ depends only on $N, \alpha_{0}, \gamma$ and $\operatorname{diam} \Omega$.

The first to prove an ABP type result for viscosity solutions of nonlinear equations was Caffarelli in his fundamental work [7]. In the case $f \in L^{\infty}$ Proposition 4.3 is due to Wang (see Theorem 3.14 and Lemma 3.19 in [41]). In its full generality, this proposition was proved in [9] (Proposition 3.3 in [9]).

We shall also make use of the following weak Harnack inequality for scalar equations (see Corollary 4.14 in [41], and Section 4.6 in [41] for more general equations). Another general result for parabolic equations was stated in [13, pp. 2022-2025].

Proposition 4.4. Let $w \in C(\Omega)$ be a non-negative solution of the scalar inequality

$$
\mathcal{L}^{-}\left(D^{2} w, D w\right)-c w \leqslant f \quad \text { in } B_{2} \subset \Omega,
$$

where $c \in L^{\infty}\left(B_{2}\right)$, with $0 \leqslant c(x) \leqslant v$ a.e. in $B_{2}$, and $f \in L^{N}\left(B_{2}\right)$. Then there exists $p=p\left(N, \alpha_{0}, \gamma, v\right)>0$ such that

$$
|w|_{p, 1} \leqslant C_{\#}\left(\inf _{B_{1}} w+\|f\|_{L^{N}\left(B_{2}\right)}\right),
$$

where $C_{\#}=C_{\#}\left(N, \alpha_{0}, \gamma, v\right)$. In particular, if $f \equiv 0$ then either $w$ is strictly positive or $w \equiv 0$ (strong maximum principle).

Remark. In fact, this proposition was proved in [41] in the particular case $f \in L^{\infty}$, but extension to $L^{N}$ is straightforward, since the proof in [41] relies only on the ABP inequality, which holds true for right-hand sides in $L^{N}$ (see Proposition 4.3 above).

The following existence result for extremal operators will be useful in the sequel.

Proposition 4.5. Let $c, f \in L^{\infty}\left(B_{2}\right)$, and $0 \leqslant c \leqslant v$ a.e. in $B_{2}$. Then there exists a unique solution $w \in$ $W_{\text {loc }}^{2, N}\left(B_{2}\right) \cap C\left(\overline{B_{2}}\right)$ of the following problem

$$
\left\{\begin{array}{l}
-\mathcal{L}^{-}\left(D^{2} w, D w\right)+c w=f \quad \text { a.e. in } B_{2}, \\
w=0 \quad \text { on } \partial B_{2} .
\end{array}\right.
$$

Moreover, $w \in W_{\mathrm{loc}}^{2, p}\left(B_{2}\right)$ for all $p<\infty$, and one has the interior estimate

$$
\|w\|_{W^{2, p}\left(B_{1}\right)} \leqslant C\left(\|w\|_{L^{\infty}\left(B_{2}\right)}+\|f\|_{L^{N}\left(B_{2}\right)}\right)
$$

where $C=C\left(N, \alpha_{0}, \gamma, \nu, p\right)$.

Proof. When $c \equiv 0$ this result was proved in [9] (Corollary 3.10 in that paper). Exactly the same proof works for $c \geqslant 0$, since the authors use Theorem 17.17 in [22] and the ABP estimate, which both hold when $c \geqslant 0$. 
Finally, we prove two lemmas concerning sums and products of viscosity solutions and test functions.

Lemma 4.2. Suppose the linear operator

$$
L_{0}=\sum_{i, j=1}^{N} a_{i j}(x) \frac{\partial^{2}}{\partial x_{i} \partial x_{j}}+\sum_{i=1}^{N} b_{i}(x) \frac{\partial}{\partial x_{i}}
$$

is uniformly elliptic in $\Omega$, and suppose $L_{0}$ has bounded measurable coefficients. Let $f, g \in L^{N}(\Omega)$.

(a) Let $w \in C(\Omega)$ and $\psi \in W_{\mathrm{loc}}^{2, N}(\Omega)$ satisfy $L_{0} w \geqslant f$ in $\Omega$ and $L_{0} \psi \geqslant g$ a.e. in $\Omega$. Then

$$
L_{0}(w+\psi) \geqslant f+g \text { in } \Omega .
$$

(b) Let $w \in C(\Omega)$ satisfy $L_{0} w \geqslant f$ in $\Omega$ and $\psi \in W_{\text {loc }}^{2, p}(\Omega) \cap C(\bar{\Omega}), p>N$, be strictly positive in $\bar{\Omega}$. Define $\tilde{w}=w / \psi$ and $\tilde{f}=f / \psi$. Then we have

$$
\tilde{L} \widetilde{w} \geqslant \tilde{f} \text { in } \Omega \text {, }
$$

where $\tilde{L}$ is defined by

$$
\tilde{L}=L_{0}+2 \sum_{i, j=1}^{N} a_{i j}(x) \frac{\partial_{i} \psi(x)}{\psi(x)} \frac{\partial}{\partial x_{j}}+\frac{L_{0} \psi(x)}{\psi(x)} .
$$

Proof. Suppose (28) does not hold. Then, by Definition 4.1, we can find an open set $\mathcal{O} \subset \Omega$ (we can assume $\mathcal{O} \Subset \Omega), \varepsilon>0, x_{0} \in \mathcal{O}$ and a function $\varphi \in W^{2, N}(\mathcal{O})$ such that

$$
\begin{aligned}
L_{0} \varphi & \leqslant f+g-\varepsilon \\
& \leqslant f+L_{0} \psi-\varepsilon \quad \text { a.e. in } \mathcal{O},
\end{aligned}
$$

$\varphi \geqslant w+\psi$ in $\mathcal{O}$ and $\varphi\left(x_{0}\right)=w\left(x_{0}\right)+\psi\left(x_{0}\right)$. Since $\psi \in W^{2, N}(\mathcal{O})$, the function $\varphi-\psi$ is a test function for the equation $L_{0} w \geqslant f$. However, $L_{0}(\varphi-\psi) \leqslant f-\varepsilon$ is a contradiction with this equation.

Next, suppose (b) is false. Then we can find an open set $\mathcal{O} \Subset \Omega, \varepsilon>0, x_{0} \in \mathcal{O}, \varphi \in W^{2, N}(\mathcal{O})$ such that $\varphi \geqslant \widetilde{w}$ in $\mathcal{O}, \varphi\left(x_{0}\right)=\widetilde{w}\left(x_{0}\right)$, and

$$
\tilde{L} \varphi \leqslant \tilde{f}-\varepsilon \quad \text { a.e. in } \mathcal{O} .
$$

A simple computation transforms (30) into

$$
\begin{aligned}
L_{0}(\varphi \psi) & \leqslant f-\varepsilon \psi \\
& \leqslant f-\varepsilon_{1} \quad \text { a.e. in } \mathcal{O},
\end{aligned}
$$

where $\varepsilon_{1}=\varepsilon \min _{\mathcal{O}} \psi>0$. Since $\varphi \psi \in W^{2, N}(\mathcal{O})\left(W^{2, N}\right.$ is an algebra), $\varphi \psi \geqslant w$ in $\mathcal{O}, \varphi \psi\left(x_{0}\right)=w\left(x_{0}\right)$, we obtain a contradiction with $L_{0} w \geqslant f$.

Lemma 4.3. Let $w \in C(\Omega)$ satisfy $\mathcal{L}^{+}\left(D^{2} w, D w\right) \geqslant f$ in $\Omega$ and suppose $\psi \in W_{\text {loc }}^{2, p}(\Omega) \cap C(\bar{\Omega}), p>N$, is strictly positive in $\bar{\Omega}$. Then $\bar{w}=w / \psi$ satisfies the inequation

$$
\mathcal{M}^{+}\left(D^{2} \bar{w}\right)+\left(\gamma+2 \alpha_{0}^{-1} \sqrt{N} \frac{|D \psi|}{\psi}\right)|D \bar{w}|+\left(\frac{\mathcal{M}^{+}\left(D^{2} \psi\right)+\gamma|D \psi|}{\psi}\right) \bar{w} \geqslant \frac{f}{\psi} .
$$

Proof. Suppose first $w \in W_{\text {loc }}^{2, N}(\Omega)$ so that $\mathcal{L}^{+}\left(D^{2} w, D w\right) \geqslant f$ is satisfied a.e. in $\Omega$. We have

$$
D w=\psi D \bar{w}+\bar{w} D \psi, \quad D^{2} w=\psi D^{2} \bar{w}+2 D \psi \otimes D \bar{w}+\bar{w} D^{2} \psi
$$


It is understood here and in the sequel that $\otimes$ denotes the symmetric tensor product, i.e., if $X, Y \in \mathbb{R}^{N}$ then $X \otimes Y=$ $\frac{1}{2}\left(x_{i} y_{j}+x_{j} y_{i}\right)_{i, j}$. By putting (32) into $\mathcal{L}^{+}\left(D^{2} w, D w\right) \geqslant f$ and by using (18) we obtain the statement of the lemma. Note that $\operatorname{tr}(A(X \otimes Y)) \leqslant|A||X \otimes Y| \leqslant \sqrt{N} \alpha_{0}^{-1}|X||Y|$, where $A$ is a matrix whose eigenvalues lie in $\left[\alpha_{0}, \alpha_{0}^{-1}\right]$, and $|A|:=\sqrt{\operatorname{tr}\left(A^{\mathrm{T}} A\right)}$. If $u$ is only continuous we proceed as in the proof of part (b) of the previous lemma.

Remark. In the same way we can prove that $\mathcal{L}^{-}\left(D^{2} w, D w\right) \leqslant f$ implies

$$
\mathcal{M}^{-}\left(D^{2} \bar{w}\right)-\left(\gamma+2 \alpha_{0}^{-1} \sqrt{N} \frac{|D \psi|}{\psi}\right)|D \bar{w}|+\left(\frac{\mathcal{M}^{-}\left(D^{2} \psi\right)-\gamma|D \psi|}{\psi}\right) \bar{w} \leqslant \frac{f}{\psi} .
$$

\section{Proof of the ABP estimate}

This section is concerned with the proof of Theorem 3.1. Recall that $u$ satisfies the system

$$
-\mathcal{L}^{+}\left(D^{2} u_{i}, D u_{i}\right)-c_{i}(u, x) \leqslant f_{i}(x), \quad i=1, \ldots, n .
$$

The first lemma permits us to linearize the zero-order terms in this system.

Lemma 5.1. Suppose $c=\left(c_{1}, \ldots, c_{n}\right): \mathbb{R}^{n} \times \Omega \rightarrow \mathbb{R}^{n}$ satisfies $(\mathrm{H} 0)$, (H3) and either $(\mathrm{H} 5)$ or $(\mathrm{H} 6)$. Then there exists $M \in L^{\infty}\left(\mathbb{R}^{n} \times \Omega, \mathcal{M}_{n}(\mathbb{R})\right)$, with $M=\left(m_{i j}\right)$, such that

$$
c(u, x)=M(u, x) u
$$

satisfying

$$
m_{i j}(u, x) \geqslant 0 \quad \forall i \neq j, i, j \in\{1, \ldots, n\},
$$

for all $u \in \mathbb{R}^{n}$ and a.e. $x \in \Omega$. In addition,

$$
\sum_{j=1}^{n} m_{i j}(u, x) \leqslant 0, \quad \forall i \in\{1, \ldots, n\},
$$

in case (H5), or

$$
m_{i j}(u, x) \leqslant \bar{m}_{i j}, \quad \forall i, j \in\{1, \ldots, n\},
$$

in case (H6) holds.

Proof. Because of (H0) the function $h(s, u, x)=\nabla_{u} c(s u, x)$ belongs to $L^{1}\left((0,1) \times B_{R} \times \Omega\right)$, for any $B_{R} \subset \mathbb{R}^{n}$, $R<\infty$ (even if $c$ is only locally Lipschitz un $u$ ). By Fubini's theorem the function

$$
M(u, x)=\int_{0}^{1} \nabla_{u} c(s u, x) \mathrm{d} s
$$

is measurable on $\mathbb{R}^{n} \times \Omega$. By (H0) $M \in L^{\infty}\left(\mathbb{R}^{n} \times \Omega, \mathcal{M}_{n}(\mathbb{R})\right)$. Further, $M$ clearly satisfies (33)-(35), and (36) for a.e. $(u, x)$.

Now for a.e. $x \in \Omega$ the matrix $M(u, x)$ is well defined for all $u \in \mathbb{R}^{n} \backslash Z_{x}$ with all the desired properties, where $Z_{x} \subset \mathbb{R}^{n}$ is a set of $n$-dimensional measure zero. It remains to define $M$ on $Z_{x}$. To this aim, for any $u \in Z_{x}$ we take $u_{k} \in \mathbb{R}^{n} \backslash Z_{x}$ such that $u_{k} \rightarrow u$ and observe that the sequence $M\left(u_{k}, x\right)$ is bounded in $\mathcal{M}_{n}(\mathbb{R})$. We can thus define $M(u, x)$ as (any) limit of a subsequence of $M\left(u_{k}, x\right)$, and observe that properties (33)-(35), and (36) are still satisfied at the limit.

This completes the proof of Lemma 5.1. 
We infer from (H1), (2) and Lemma 5.1 that

$$
\mathcal{M}^{+}\left(D^{2} u_{i}\right)+\gamma\left|D u_{i}\right|+\sum_{j=1}^{n} m_{i j}(u(x), x) u_{j} \geqslant-f_{i}^{+},
$$

for $i=1, \ldots, n$. We set $m_{i j}(x)=m_{i j}(u(x), x)$.

We claim that the same inequality is satisfied by the positive parts of the functions $u_{i}$.

Claim 5.1. We have

$$
\mathcal{M}^{+}\left(D^{2} u_{i}^{+}\right)+\gamma\left|D u_{i}^{+}\right|+\sum_{j=1}^{n} m_{i j}(x) u_{j}^{+} \geqslant-f_{i}^{+} .
$$

Proof. By (34) $m_{i j} u_{j} \leqslant m_{i j} u_{j}^{+}$, for $i \neq j$. Hence both $v=u_{i}$ and $v=0$ satisfy the inequality

$$
\mathcal{M}^{+}\left(D^{2} v\right)+\gamma|D v|+m_{i i}(x) v \geqslant-f_{i}^{+}-\sum_{j \neq i}^{n} m_{i j}(x) u_{j}^{+} .
$$

Hence, by Proposition 4.1, $u_{i}^{+}=\max \left(u_{i}, 0\right)$ satisfies the same inequation.

We are now ready to prove the ABP inequality in case (H5) is satisfied. By the previous considerations we can restrict ourselves to a system of type (39), with $m_{i j}$ satisfying (34) and (35). The basic idea of the proof is to show that the function

$$
v(x)=u_{1}^{+} \vee \cdots \vee u_{n}^{+}(x)
$$

satisfies a scalar elliptic inequation, and then apply the scalar ABP estimate to $v$.

Lemma 5.2. Under (H5) we have

$$
-\mathcal{L}^{+}\left(D^{2} v, D v\right) \leqslant f_{1}^{+} \vee \cdots \vee f_{n}^{+} \quad \text { in } \Omega .
$$

Proof. Suppose for contradiction that there are an open set $\mathcal{O} \Subset \Omega$, a point $x_{0} \in \mathcal{O}, \varepsilon>0$, and a function $\varphi \in W^{2, N}(\mathcal{O})$ such that $v \leqslant \varphi$ in $\mathcal{O}, v\left(x_{0}\right)=\varphi\left(x_{0}\right)$, and

$$
-\mathcal{L}^{+}\left(D^{2} \varphi, D \varphi\right) \geqslant f_{1}^{+} \vee \cdots \vee f_{n}^{+}+\varepsilon>0 \quad \text { a.e. in } \mathcal{O} .
$$

We have to show that (42) is impossible. Fix $k \in\{1, \ldots, n\}$ such that $u_{k}^{+}\left(x_{0}\right)=v\left(x_{0}\right)$. Then

$$
\varphi \geqslant u_{k}^{+} \geqslant 0 \text { in } \mathcal{O} \text { and } \varphi\left(x_{0}\right)=u_{k}^{+}\left(x_{0}\right) .
$$

We distinguish two cases. First, if $\varphi\left(x_{0}\right)=0$, we see that $\varphi$ attains a local minimum at $x_{0}$. Then we apply the scalar strong maximum principle (see Proposition 4.4) to (42) and obtain $\varphi \equiv 0$ in $\mathcal{O}$. This contradicts (42).

Second, if $\varphi\left(x_{0}\right)>0$, we have, by (34), (35), (42) and (H0)

$$
\begin{aligned}
-\mathcal{L}^{+}\left(D^{2} \varphi, D \varphi\right) & \geqslant\left(\sum_{j=1}^{n} m_{k j}\right) u_{k}^{+}+f_{k}^{+}+\varepsilon \\
& \geqslant \sum_{j=1}^{n} m_{k j} u_{j}^{+}+f_{k}^{+}+\frac{\varepsilon}{2} \quad \text { a.e. in } \mathcal{O}_{1},
\end{aligned}
$$


where $\mathcal{O}_{1} \subset \mathcal{O}$ is an open neighbourhood of $x_{0}$ in which

$$
u_{k}>0 \text { and } u_{k} \geqslant u_{j}^{+}-\varepsilon / 2 v,
$$

for all $j \in\{1, \ldots, n\}\left(\mathcal{O}_{1}\right.$ exists, since $\left.u_{k}\left(x_{0}\right)=u_{1} \vee \cdots \vee u_{n}\left(x_{0}\right)>0\right)$.

By (39) $u_{k}$ is a (viscosity) solution of

$$
-\mathcal{L}^{+}\left(D^{2} u_{k}^{+}, D u_{k}^{+}\right) \leqslant \sum_{j=1}^{n} m_{k j} u_{j}^{+}+f_{k}^{+},
$$

which is a contradiction with (43) and (45). Lemma 5.2 is proved.

Now we can apply Proposition 4.3 to (41). We obtain

$$
\sup _{\Omega} v \leqslant \sup _{\partial \Omega} v+C_{*}\left\|f_{1}^{+} \vee \cdots \vee f_{n}^{+}\right\|_{L^{N}(\Omega)},
$$

which gives part (b) of Theorem 3.1.

Remark. If the functions $c_{i}$ are supposed to be only locally Lipschitz the above proof remains the same, if we replace $v$ in (46) by the essential supremum of all $\sum_{j}\left|m_{i j}(x)\right|$ in a neighbourhood of $x_{0}$.

We are going to show that if our system satisfies assumption (H6) then we can introduce a change of functions so that the transformed system satisfies (H5). So let us assume (H6), which says $m_{i j}(x) \leqslant \bar{m}_{i j}$ for a.e. $x$, where $\bar{M}=\left(\bar{m}_{i j}\right)$ is a negative semi-definite matrix. First we perturb the system in order to make the zero order matrix negative definite. To this purpose, we set $\bar{u}_{i}=u_{i}^{+} / \psi$, where $\psi$ is the solution of

$$
\left\{\begin{array}{l}
\mathcal{M}^{+}\left(D^{2} \psi\right)+\gamma|D \psi|=-1 \quad \text { in } \widetilde{\Omega}, \\
\psi=0 \text { on } \partial \widetilde{\Omega} \\
\psi \in W_{\text {loc }}^{2, p}(\widetilde{\Omega}) \cap C(\overline{\widetilde{\Omega}}) \quad \forall p \in(1,+\infty)
\end{array}\right.
$$

(this problem is solvable, see Proposition 4.5 and (20)), where $\widetilde{\Omega}$ is such that $\Omega \Subset \widetilde{\Omega}$. By the scalar maximum principle $\psi>0$ in $\widetilde{\Omega}$. By using the scalar ABP inequality, Lemma 4.1 , and a theorem by Krylov which we state later (Theorem 7.1 in Section 7), one can see that $\bar{C} \geqslant \psi \geqslant \bar{c}>0$ in $\Omega$, where $\bar{C}$ and $\bar{c}$ are constants which depend only on $N, \alpha_{0}, \gamma$, and $\operatorname{diam}(\Omega)$.

By (H6), (39) and Lemma $4.3 \bar{u}$ satisfies the system of inequations

$$
\mathcal{M}^{+}\left(D^{2} \bar{u}_{i}\right)+\left(\gamma+2 \alpha_{0}^{-1} \sqrt{N} \frac{\|D \psi\|_{\infty}}{\inf _{\Omega} \psi}\right)\left|D \bar{u}_{i}\right|+\sum_{j}\left(\bar{m}_{i j}-\varepsilon \delta_{i j}\right) \bar{u}_{j} \geqslant-\frac{f_{i}^{+}}{\psi},
$$

where

$$
\varepsilon=-\frac{\mathcal{M}^{+}\left(D^{2} \psi\right)+\gamma|D \psi|}{\sup _{\Omega} \psi}=\frac{1}{\sup _{\Omega} \psi}>0 .
$$

Set $M_{\varepsilon}=\varepsilon I-\bar{M}\left(M_{\varepsilon}\right.$ is positive definite), and let $\xi \in \mathbb{R}^{n}$ be the solution of the linear system

$$
M_{\varepsilon} \xi=(1, \ldots, 1)
$$


We claim that assumptions (H3) and (H6) imply $\xi_{i}>0$ for all $i$. In order to prove this we suppose first that $M_{\varepsilon}$ is symmetric. It is well-known that (H6) implies that all principal minors of $M_{\varepsilon}$ are positive. We are going to use an algebraic lemma from [15] (Lemma 2.2 in that paper), the statement of which we give for readers' convenience.

Lemma 5.3 (de Figueiredo-Mitidieri). Let $M=\left(m_{i j}\right) \in \mathcal{M}_{n}(\mathbb{R})$ be a matrix such that $m_{i j} \leqslant 0$ for $i \neq j$, and $\operatorname{det}\left(\left(m_{i j}\right)_{i, j=1}^{k}\right)>0$, for every $k \in\{1, \ldots, n\}$. Then

$$
(-1)^{i+j} \operatorname{det} M^{i j}>0
$$

where $M^{i j}$ is the submatrix of $M$ obtained by dropping its $i$-th line and $j$-th column.

It follows from this lemma that $(-1)^{i+j} \operatorname{det}\left(M_{\varepsilon}^{i j}\right)>0$. By Cramer's rule

$$
\xi_{i}=\operatorname{det}\left(M_{\varepsilon}^{-1}\right) \sum_{j=1}^{n}(-1)^{i+j} \operatorname{det}\left(M_{\varepsilon}^{i j}\right)>0 .
$$

If $M_{\varepsilon}$ is not symmetric we use the following elementary algebraic lemma.

Lemma 5.4. If $A$ is a positive definite matrix then

$$
\operatorname{det}(A) \geqslant \operatorname{det}\left(\frac{A+A^{\mathrm{T}}}{2}\right)>0 .
$$

Lemma 5.4 implies that all principal minors of $M_{\varepsilon}$ are positive, even if $M_{\varepsilon}$ is not symmetric. For completeness, we give a proof of Lemma 5.4 at the end of this section.

We can now finish the proof of the ABP estimate. We set $\bar{u}_{i}=\xi_{i} \tilde{u}_{i}$. These transformed functions clearly satisfy

$$
\mathcal{M}^{+}\left(D^{2} \tilde{u}_{i}\right)+\gamma^{\prime}\left|D \tilde{u}_{i}\right|+\frac{1}{\xi_{i}} \sum_{j} \tilde{d}_{i j} \tilde{u}_{j} \geqslant-\widetilde{f_{i}^{+}},
$$

where

$$
\begin{aligned}
& \tilde{d}_{i j}=\left(\bar{m}_{i j}-\varepsilon \delta_{i j}\right) \xi_{j}, \quad \widetilde{f_{i}^{+}}=\frac{f_{i}^{+}}{\xi_{i} \inf _{\Omega} \psi}, \\
& \gamma^{\prime}=\left(2 \alpha_{0}^{-1} \sqrt{N} \frac{\|D \psi\|_{\infty}}{\inf _{\Omega} \psi}+\gamma\right) .
\end{aligned}
$$

By (49), the zero-order matrix in (50) satisfies

$$
\frac{1}{\xi_{i}} \sum_{j=1}^{n} \tilde{d}_{i j}=-\frac{1}{\xi_{i}}<0
$$

for all $i$, i.e., assumption (H5). Therefore we can apply to (50) the ABP inequality we already proved. Since $\tilde{u}_{i}=u_{i}^{+} /\left(\xi_{i} \psi\right)$ we get

$$
\sup _{\Omega}\left(u_{1} \vee \cdots \vee u_{n}\right) \leqslant \frac{\sup _{i, x} \xi_{i} \psi(x)}{\inf _{i, x} \xi_{i} \psi(x)}\left(\sup _{\partial \Omega}\left(u_{1}^{+} \vee \cdots \vee u_{n}^{+}\right)+C\left\|f^{+}\right\|_{L^{N}(\Omega)}\right) .
$$

Theorem 3.1 is proved.

We obtain the result in Theorem 3.1bis by setting $v=-u$ in Theorem 3.1, by using (H2), and by noting that $\tilde{g}_{i}(u, x)=-g_{i}(-u, x)$ has the same properties as $g_{i}$ (namely, satisfies (H0) and (H3)). 
Proof of Lemma 5.4. The lemma follows from the following more general fact.

Claim 5.2. Let $B \in \mathcal{M}_{n}(\mathbb{R})$ be a positive definite symmetric matrix and let $R \in \mathcal{M}_{n}(\mathbb{R})$ be a skew-symmetric matrix $\left(R^{\mathrm{T}}=-R\right)$. Then

$$
\operatorname{det}(B+x R)=\sum_{k=0}^{[n / 2]} a_{k} x^{2 k},
$$

where $a_{k} \geqslant 0$ if $k \geqslant 1$, and $a_{0}=\operatorname{det}(B)>0$.

Lemma 5.4 follows by taking $B=\left(A+A^{\mathrm{T}}\right) / 2, R=\left(A-A^{\mathrm{T}}\right) / 2$, and $x=1$ in the claim (note that $A$ and $B$ are matrices of the same quadratic form).

Proof of Claim 5.2. First we note that for any skew-symmetric matrix $D$ the characteristic polynomial of $D$ can be written in the following form

$$
\operatorname{det}(\lambda I-D)=\lambda^{p}\left(\lambda^{2}+\beta_{1}\right) \cdots\left(\lambda^{2}+\beta_{m}\right)
$$

where $\beta_{i}>0, i=1, \ldots, m, p+2 m=n$. Indeed, if $\mu \in \mathbb{C}$ and $v \in \mathbb{C}^{n}$ are such that $R v=\mu v$, the equality $(R v, v)=\left(v, R^{\mathrm{T}} v\right)$ implies $\mu=-\bar{\mu}$.

Next, we write

$$
\begin{aligned}
\operatorname{det}(B+x R) & =\operatorname{det}(B) \cdot x^{n} \cdot \operatorname{det}\left(B^{-1} R+\frac{1}{x} I\right) \\
& =\operatorname{det}(B) \cdot x^{n} \cdot(-1)^{n} \operatorname{det}(\lambda I-S R),
\end{aligned}
$$

where $\lambda=-1 / x$ and $S=B^{-1}$ is a positive definite symmetric matrix. We set $D=S^{1 / 2} R S^{1 / 2}$. Then $D$ is a skewsymmetric matrix, and the eigenvalues of $D$ and $S R$, counted with their multiplicities, are the same (if $v \in \mathbb{C}^{n}$ is an eigenvector of $S R$ corresponding to the eigenvalue $\mu$ and $w$ solves $S^{1 / 2} w=v$, then $w \in \mathbb{C}^{n}$ is an eigenvector of $D$ corresponding to the same eigenvalue). Equalities (54) and (55) then yield

$$
\operatorname{det}(B+x R)=\operatorname{det}(B)\left(\beta_{1} x^{2}+1\right) \cdots\left(\beta_{m} x^{2}+1\right),
$$

which gives the desired result.

\section{Proof of the local maximum principle}

This section is devoted to the proof of Proposition 3.1. From now on, we suppose that $R=1$ and $B_{1}$ is centered at $y_{0}=0$, the general case being obtained by means of the coordinate transformation $x \rightarrow\left(x-y_{0}\right) / R$.

First, we claim that the function $u_{k}^{+}$satisfies the inequation

$$
-\mathcal{L}^{+}\left(D^{2} u_{k}^{+}, D u_{k}^{+}\right) \leqslant v u_{1}^{+} \vee \cdots \vee u_{n}^{+}+f^{+} \text {in } B_{3}
$$

(in the viscosity sense), for all $k=1, \ldots, n$. Recall that $f=f_{1} \vee \cdots \vee f_{n}$.

Proof of (56). Set $v=u_{1}^{+} \vee \cdots \vee u_{n}^{+}$. Suppose (56) is false. Then, by Definition 4.1, there exist an open set $\mathcal{O} \Subset B_{3}$, $x_{0} \in \mathcal{O}, \varphi \in W^{2, N}(\mathcal{O})$, and a real number $\varepsilon>0$ such that $v \leqslant \varphi$ in $\mathcal{O}, v\left(x_{0}\right)=\varphi\left(x_{0}\right)$ and

$$
-\mathcal{L}^{+}\left(D^{2} \varphi, D \varphi\right) \geqslant v v+f^{+}+\varepsilon \quad \text { a.e. in } \mathcal{O} \text {. }
$$

Observe that the hypotheses of Proposition 3.1 imply, by Lemma 5.1, 


$$
\begin{aligned}
-\mathcal{L}^{+}\left(D^{2} u_{k}, D u_{k}\right) & \leqslant \sum_{j \neq k} m_{k j}(x) u_{j}^{+}(x)+m_{k k}(x)\left(u_{k}^{+}-u_{k}^{-}\right) \\
& \leqslant v v+m_{k k}^{-}(x) u_{k}^{-}+f^{+} \quad \text { in } \Omega
\end{aligned}
$$

for all $k=1, \ldots, n$. We then proceed as in the proof of the ABP estimate, Lemma 5.2. If $v\left(x_{0}\right)=\varphi\left(x_{0}\right)>0$, we take $k$ such that $v\left(x_{0}\right)=u_{k}\left(x_{0}\right)$ and obtain a contradiction with (57) and (58) in some open set $\mathcal{O}_{1} \subset \mathcal{O}$, where $u_{k}>0$ (so that $m_{k k}^{-} u_{k}^{-} \equiv 0$ in $\mathcal{O}_{1}$ ). If $\varphi\left(x_{0}\right)=0, \varphi$ attains a local minimum at $x_{0}$ and we obtain a contradiction with the strong maximum principle, as in the proof of Lemma 5.2.

Using the fact that the maximum of subsolutions is a subsolution (Proposition 4.1), we obtain from (56)

$$
-\mathcal{L}^{+}\left(D^{2} v, D v\right) \leqslant v v+f^{+} \quad \text { in } B_{3}
$$

in the viscosity sense. In order to obtain the conclusion of Proposition 3.1 we use Proposition 4.3, combined with the localization argument in the proof of Theorem 9.20 in [22]. We are going to show that this argument can be adapted to our situation. Since it relies on a cut-off procedure and pointwise estimates, for the differential relations to make sense we shall use a regularized version of $v$, namely its sup-convolution, defined by

$$
v_{\varepsilon}(x)=\sup _{y \in B_{5 / 2}}\left\{v(y)-\frac{1}{2 \varepsilon}|x-y|^{2}\right\} .
$$

Let us suppose first that $f$ is continuous in $B_{3}$. We recall the following well-known properties of the supconvolution, see [28] and [29].

\section{Lemma 6.1.}

(1) $v_{\varepsilon} \in C^{0,1}\left(B_{5 / 2}\right)$;

(2) $v_{\varepsilon} \rightarrow v$ uniformly in $\overline{B_{2}}$;

(3) $v_{\varepsilon}$ is twice differentiable a.e. in $B_{2}$;

(4) As a consequence of (59), $v_{\varepsilon}$ satisfies

$$
-\mathcal{L}^{+}\left(D^{2} v_{\varepsilon}, D v_{\varepsilon}\right) \leqslant v \widetilde{v_{\varepsilon}}+\widetilde{f_{\varepsilon}^{+}} \quad \text { a.e. in } B_{2},
$$

where

$$
\widetilde{v_{\varepsilon}}(x)=\sup _{|x-y|<\delta(\varepsilon)} v_{\varepsilon}(y), \quad \widetilde{f_{\varepsilon}^{+}}(x)=\sup _{|x-y|<\delta(\varepsilon)} f^{+}(y),
$$

with $\delta(\varepsilon)=2\left(\varepsilon\|v\|_{L^{\infty}\left(B_{5 / 2}\right)}\right)^{1 / 2}$.

We then set $w_{\varepsilon}=\eta_{\varepsilon} v_{\varepsilon}$, where

$$
\eta_{\varepsilon}(x)=(2-\delta(\varepsilon))^{-2 \beta}\left((2-\delta(\varepsilon))^{2}-|x|^{2}\right)^{\beta},
$$

for some $\beta \geqslant 2$. For simplicity of notation, we write $\eta$ instead of $\eta_{\varepsilon}$.

By (16)-(18) and (61), we have

$$
\begin{aligned}
-\mathcal{L}^{+}\left(D^{2} w_{\varepsilon}, D w_{\varepsilon}\right) & \leqslant-\mathcal{M}^{+}\left(\eta D^{2} v_{\varepsilon}\right)-2 \mathcal{M}^{-}\left(D \eta \otimes D v_{\varepsilon}\right)-\mathcal{M}^{-}\left(v_{\varepsilon} D^{2} \eta\right)-\gamma\left|\eta D v_{\varepsilon}+v_{\varepsilon} D \eta\right| \\
& \leqslant \eta\left(-\mathcal{M}^{+}\left(D^{2} v_{\varepsilon}\right)-\gamma\left|D v_{\varepsilon}\right|\right)+2 \mathcal{M}^{+}\left((-D \eta) \otimes D v_{\varepsilon}\right)+\mathcal{M}^{+}\left(-v_{\varepsilon} D^{2} \eta\right)+\gamma v_{\varepsilon}|D \eta| \\
& \leqslant \nu \eta \widetilde{v_{\varepsilon}}+\widetilde{f_{\varepsilon}^{+}}+2 \alpha_{0}^{-1} \sqrt{N}\left(\left|D \eta \| D v_{\varepsilon}\right|+\left|D^{2} \eta\right| v_{\varepsilon}\right)+\gamma v_{\varepsilon}|D \eta|
\end{aligned}
$$


a.e. in $B_{2-\delta(\varepsilon)}$ (see also the proof of Lemma 4.3). Let us denote by $H$ the right-hand side in the last inequality. By using Proposition 4.3 we obtain

$$
\sup _{B_{2-\delta(\varepsilon)}} w_{\varepsilon} \leqslant C_{*}\|H\|_{L^{N}\left(\Gamma_{\varepsilon}^{+}\right)},
$$

where $\Gamma_{\varepsilon}^{+}$is the upper contact set of $w_{\varepsilon}$, see Proposition 4.3. By a concavity argument we get, as in the proof of Theorem 9.20 in [22],

$$
\left|D v_{\varepsilon}\right| \leqslant C(\beta) \eta^{-1 / \beta} v_{\varepsilon}
$$

on $\Gamma_{\varepsilon}^{+}$. Since

$$
|D \eta| \leqslant C \eta^{1-1 / \beta}, \quad\left|D^{2} \eta\right| \leqslant C \eta^{1-2 / \beta},
$$

we see that

$$
H \leqslant \eta \nu \widetilde{v_{\varepsilon}}+C\left(\eta^{-2 / \beta} w_{\varepsilon}+f_{\varepsilon}^{+}\right)
$$

a.e. on $\Gamma_{\varepsilon}^{+}$.

Here and in the sequel $C$ denotes a constant which depends on the appropriate quantities and may vary from line to line.

Then we follow the lines of the proof of Theorem 9.20 in [22] (in particular the last three inequalities in this proof) to infer from (63) and (64)

$$
\sup _{B_{2-\delta(\varepsilon)}} w_{\varepsilon} \leqslant C\left(\left\|\eta \widetilde{v_{\varepsilon}}\right\|_{L^{N}\left(B_{2}\right)}+\left\|v_{\varepsilon}\right\|_{L^{p}\left(B_{2}\right)}+\left\|\widetilde{f_{\varepsilon}^{+}}\right\|_{L^{N}\left(B_{2}\right)}\right),
$$

where $C$ does not depend on $\varepsilon$. By interpolation this implies

$$
\sup _{B_{2-\delta(\varepsilon)}} \eta v_{\varepsilon}-\frac{1}{2} \sup _{B_{2}} \eta \widetilde{v_{\varepsilon}} \leqslant C\left(\left\|\widetilde{v_{\varepsilon}}\right\|_{L^{p}\left(B_{2}\right)}+\left\|v_{\varepsilon}\right\|_{L^{p}\left(B_{2}\right)}+\left\|\widetilde{f_{\varepsilon}^{+}}\right\|_{L^{N}\left(B_{2}\right)}\right) .
$$

Note that, by (2) in Lemma $6.1 \widetilde{v_{\varepsilon}} \rightarrow v$ uniformly on $\overline{B_{2}}$.

By letting $\varepsilon \rightarrow 0$ we obtain the desired result.

Finally we have to remove the continuity assumption on $f$. This can be done through a (standard) approximation argument. We take a solution of the problem

$$
\left\{\begin{array}{l}
-\mathcal{L}^{-}\left(D^{2} \psi^{j}, D \psi^{j}\right)=f^{j}-f^{+} \text {in } B_{2}, \\
\psi^{j}=0 \text { on } \partial B_{2}, \\
\psi^{j} \in W_{\text {loc }}^{2, N}(\Omega) \cap C\left(\overline{B_{2}}\right)
\end{array}\right.
$$

(see Proposition 4.5), where $f^{j} \in C^{\infty}\left(\overline{B_{2}}\right), f^{j} \rightarrow f^{+}$in $L^{N}\left(B_{2}\right)$.

Set $v^{j}=v+\psi^{j}$. The scalar ABP inequality (Proposition 4.3), applied to (67), implies $\psi^{j} \rightarrow 0$ uniformly in $B_{2}$. Then by (18)

$$
\mathcal{L}^{+}\left(D^{2} v, D v\right) \leqslant \mathcal{L}^{+}\left(D^{2} v^{j}, D v^{j}\right)-\mathcal{L}^{-}\left(D^{2} \psi^{j}, D \psi^{j}\right)=\mathcal{L}^{+}\left(D^{2} v^{j}, D v^{j}\right)+f^{j}-f^{+} .
$$

Note that this is valid in the viscosity sense since $\psi^{j}$ has the regularity of a test function $\left(\psi^{j} \in W_{\text {loc }}^{2, N}(\Omega)\right)$. Now, by (59)

$$
-\mathcal{L}^{+}\left(D^{2} v^{j}, D v^{j}\right) \leqslant v v+f^{j}=v v^{j}+\overline{f^{j}}
$$

where $\overline{f^{j}}=f^{j}+v\left(v-v^{j}\right)$. Note that $\overline{f^{j}} \in C\left(\overline{B_{2}}\right)$ and $\overline{f^{j}} \rightarrow f^{+}$in $L^{N}\left(B_{2}\right)$.

Applying the result we already proved ( $f^{j}$ is continuous) to (69) with $v^{j}$ instead of $v$ and sending $j \rightarrow \infty$ in the final inequality concludes the proof. 


\section{Proof of the Harnack inequality}

In this section we prove the Harnack and the weak Harnack inequalities (that is, Theorem 3.2 and Proposition 3.2) in the case when system (1) is fully coupled. We recall that we have taken $R=1$.

We shall use the following equivalent definition of full coupling by a chain (see, for instance, [11]).

Lemma 7.1. The system (1) is fully coupled in $\Omega$ if and only if for any $k, l \in\{1, \ldots, n\}$, with $k \neq l$, we can find a sequence of indices $\left\{i_{j}\right\}_{j=0}^{r}$, such that $i_{j} \neq i_{j+1}$ for all $j=0, \ldots, r-1, i_{0}=k, i_{r}=l$, and sets with positive Lebesgue measure $\omega_{i_{j} i_{j+1}} \subset \Omega$ such that

$$
\varphi_{i_{j} i_{j+1}}(t)>0 \text { for all } t>0
$$

(recall that $\varphi_{k l}$ are defined in (8)).

For each couple $i, j \in\{1, \ldots, n\}, i \neq j$, we define the function

$$
\tilde{\varphi}_{i j}(t)=\left(\kappa_{i j} \varphi_{i j}(t)-C_{*}\|f\|_{L^{N}\left(B_{3}\right)}\right)^{+},
$$

where $\kappa_{i j}=\kappa_{i j}\left(N, n, \alpha_{0}, \gamma, \nu\right.$, meas $\left.\left(\omega_{i j}\right)\right)$ are positive constants (to be defined later) and $C_{*}=C_{*}\left(N, \alpha_{0}, \gamma, \nu\right)$ is the constant from the scalar ABP inequality (Proposition 4.3).

We set

$$
\tilde{\varphi}(t)=\inf _{k \neq l} \tilde{\varphi}_{k i_{1}} \circ \tilde{\varphi}_{i_{1} i_{2}} \circ \cdots \circ \tilde{\varphi}_{i_{r-1} l}(t),
$$

where for each $(k, l), k \neq l$, the chain $\left\{i_{j}\right\}_{j=0}^{r}$ is chosen as in Lemma 7.1 above. Note that $\tilde{\varphi}$ is a Lipschitz continuous non-decreasing function of $t$, with $\tilde{\varphi}(0)=0$ in case $f \equiv 0$.

The following lemma plays a crucial role. It relates the values of the infimums of the functions $u_{i}$.

Lemma 7.2. Under the hypotheses of Theorem 3.2, suppose the indices $k$ and $l$ are such that $k \neq l$ and there exists a set $\omega_{k l} \subset B_{1}$ with meas $\left(\omega_{k l}\right)>0$, such that $\varphi_{k l}(t)>0$ for all $t>0$. Then

$$
\inf _{B_{1}} u_{k} \geqslant \tilde{\varphi}_{k l}\left(\inf _{B_{2}} u_{l}\right) \text {. }
$$

Therefore, for all $k \neq l$,

$$
\inf _{B_{1}} u_{k} \geqslant \tilde{\varphi}\left(\inf _{B_{2}} u_{l}\right)
$$

Proof. If $\inf _{B_{2}} u_{l}=0$ there is nothing to prove. So suppose $\inf _{B_{2}} u_{l}>0$. By assumption (H2) we have

$$
\mathcal{L}^{-}\left(D^{2} u_{k}, D u_{k}\right)+g_{k}(u, x) \leqslant f_{k} .
$$

By using the cooperativity assumption (H3) we get

$$
\mathcal{L}^{-}\left(D^{2} u_{k}, D u_{k}\right)+g_{k}\left(u_{k} e_{k}+u_{l} e_{l}, x\right) \leqslant f_{k},
$$

where $e_{i}$ is the $i$-th vector in the canonical basis of $\mathbb{R}^{n}$. By the assumed (uniform in $x$ ) Lipschitz continuity of $g_{k}$, together with the cooperativity assumption (H3), (76) yields

$$
\mathcal{L}^{-}\left(D^{2} u_{k}, D u_{k}\right)+g_{k}\left(\inf _{B_{2}} u_{l} e_{l}, x\right)-v u_{k} \leqslant f_{k}
$$

(recall $v$ is a Lipschitz constant for $g_{k}$ ). 
We are going to estimate $u_{k}$ from below by the solution of the following problem (see Proposition 4.5)

$$
\left\{\begin{array}{l}
\mathcal{L}^{-}\left(D^{2} w, D w\right)-v w=-g_{k}\left(\inf _{B_{2}} u_{l} e_{l}, x\right) \quad \text { in } B_{2}, \\
w \in W_{\text {loc }}^{2, p}\left(B_{2}\right) \cap C\left(\overline{B_{2}}\right), \quad \forall p \in(1,+\infty), \\
w=0 \text { on } \partial B_{2} .
\end{array}\right.
$$

Note that $g_{k}(0, x)=0$ and $g_{k}(\cdot, x) \in C^{0,1}$ imply $g_{k}(u, x) \in L^{\infty}(\Omega)$ for $u$ fixed.

We are going to infer from (77) and (78) an elliptic inequality for the function $u_{k}-w$. We use the following (essentially known) lemma.

Lemma 7.3. Let $\omega$ be a domain and suppose $w_{1}, w_{2} \in C(\bar{\omega})$ satisfy

$$
\mathcal{M}^{-}\left(D^{2} w_{1}\right)-\gamma\left|D w_{1}\right| \leqslant h \text { and } \mathcal{M}^{-}\left(D^{2} w_{2}\right)-\gamma\left|D w_{2}\right| \geqslant 0 \quad \text { in } \omega
$$

in the viscosity sense, for some $h \in L^{N}(\omega)$, and that $w_{2} \in W^{2, N}(\omega)$. Then

$$
-\mathcal{M}^{+}\left(D^{2}\left(w_{2}-w_{1}\right)\right)-\gamma\left|D\left(w_{2}-w_{1}\right)\right| \leqslant h \text { in } \omega .
$$

Proof of Lemma 7.3. Let us take $x_{0} \in \mathcal{O} \subset \omega, \varepsilon>0$ and a test function $\phi \in W^{2, N}(\mathcal{O})$ satisfying $\phi \geqslant w_{2}-w_{1}$ in $\mathcal{O}, \phi\left(x_{0}\right)=w_{2}\left(x_{0}\right)-w_{1}\left(x_{0}\right)$. Suppose for contradiction that

$$
-\mathcal{L}^{+}\left(D^{2} \phi, D \phi\right) \geqslant h+\varepsilon \text { in } \mathcal{O} \text {. }
$$

By (17), (18), (79) and (80) we get

$$
\begin{aligned}
-\mathcal{L}^{+}\left(D^{2}\left(\phi-w_{2}\right), D\left(\phi-w_{2}\right)\right) & \geqslant-\mathcal{M}^{+}\left(D^{2} \phi\right)+\mathcal{M}^{-}\left(D^{2} w_{2}\right)-\gamma|D \phi|-\gamma\left|D w_{2}\right| \\
& \geqslant-\mathcal{M}^{+}\left(D^{2} \phi\right)-\gamma|D \phi| \\
& \geqslant h+\varepsilon
\end{aligned}
$$

in $\mathcal{O}$.

Since $w_{2} \in W^{2, N}(\omega), \phi-w_{2}$ is a test function which satisfies $\phi-w_{2} \geqslant-w_{1}$ in $\mathcal{O}$, with equality at $x_{0}$. Now since $-w_{1}$ satisfies

$$
-\mathcal{L}^{+}\left(D^{2}\left(-w_{1}\right), D\left(-w_{1}\right)\right) \leqslant h
$$

we get, by Definition 4.1,

$$
-\mathcal{L}^{+}\left(D^{2}\left(\phi-w_{2}\right), D\left(\phi-w_{2}\right)\right) \ngtr h+\varepsilon
$$

in $\mathcal{O}$, which is a contradiction.

From inequalities (77), (78) and the above lemma, we deduce that the function $u_{k}-w$ satisfies the inequality

$$
-\mathcal{L}^{+}\left(D^{2}\left(w-u_{k}\right), D\left(w-u_{k}\right)\right)+v\left(w-u_{k}\right) \leqslant f_{k}
$$

in $B_{2}$. Since $w-u_{k} \leqslant 0$ on $\partial B_{2}$ the scalar ABP inequality (Proposition 4.3) implies

$$
-\inf _{B_{2}}\left(u_{k}-w\right)=\sup _{B_{2}}\left(w-u_{k}\right) \leqslant C_{*}\left\|f_{k}\right\|_{L^{N}\left(B_{2}\right)},
$$

which yields

$$
u_{k}(x) \geqslant w(x)-C_{*}\left\|f_{k}\right\|_{L^{N}\left(B_{2}\right)} \geqslant \inf _{B_{1}} w-C_{*}\left\|f_{k}\right\|_{L^{N}\left(B_{2}\right)}
$$


for all $x \in B_{1}$. Hence

$$
\inf _{B_{1}} u_{k} \geqslant \inf _{B_{1}} w-C_{*}\left\|f_{k}\right\|_{L^{N}\left(B_{2}\right)} .
$$

The point is that $\inf _{B_{1}} w$ can be estimated from below by $\varphi_{k l}\left(\inf _{B_{2}} u_{l}\right)$. Let us prove this. By Lemma 4.1 there exists an linear second order uniformly elliptic operator $\tilde{L}$ such that $\tilde{L} w=\mathcal{L}^{-}\left(D^{2} w, D w\right)$. Then (H3) and (78) yield

$$
\left\{\begin{array}{l}
\tilde{L} w-v w=-g_{k}\left(\inf _{B_{2}} u_{l} e_{l}, x\right) \leqslant 0 \quad \text { in } B_{2}, \\
\tilde{L} w-v w=-g_{k}\left(\inf _{B_{2}} u_{l} e_{l}, x\right) \leqslant-\varphi_{k l}\left(\inf _{B_{2}} u_{l}\right) \quad \text { in } \omega_{k l} \subset B_{1}, \\
w=0 \text { on } \partial B_{2} .
\end{array}\right.
$$

By the usual maximum principle $w \geqslant 0$ in $B_{2}$. We now use the following consequence of a theorem by Krylov (Theorem 12 on p. 129 in [30]), in the form which was stated in [2].

Theorem 7.1 (Krylov). Let $L_{0}$ be a linear uniformly elliptic operator with bounded measurable coefficients in the form (27), and suppose $c \in L^{\infty}\left(B_{2}\right)$. Suppose $\alpha_{0}$ is an ellipticity constant for $L_{0}$, and $\tau$ is an upper bound for the $L^{\infty}$-norms of $c$ and the first order coefficients of $L_{0}$. Let $v \in W_{\mathrm{loc}}^{2, N}\left(B_{2}\right)$ be a positive function satisfying $L_{0} v+c v \leqslant 0$ a.e. in $B_{2}$ and $L_{0} v+c v \leqslant-\rho$ a.e. in a closed subset $\omega \subset B_{2}$, for some $\rho>0$. Then there exists a constant $m>0$, depending only on $N, \alpha_{0}, \tau$, and a positive lower bound on meas $(\omega)>0$, such that

$$
\inf _{B_{1}} v \geqslant m \rho .
$$

This theorem and (87) give the following estimate from below

$$
\inf _{B_{1}} w \geqslant \kappa_{k l} \varphi_{k l}\left(\inf _{B_{2}} u_{l}\right)
$$

where $\kappa_{k l}$ is the constant from Theorem 7.1. Combining (86) and (89) yields

$$
\inf _{B_{1}} u_{k} \geqslant \tilde{\varphi}_{k l}\left(\inf _{B_{2}} u_{l}\right) \text {. }
$$

This proves (73) in Lemma 7.2. Finally, we take a sequence $\left\{i_{j}\right\}_{j=0}^{r}$ as in Lemma 7.1 and a sequence of nested balls $\left\{B_{\alpha_{j}}\right\}_{j=0}^{r}, \alpha_{j}=1+j / r$. Then, as above,

$$
\tilde{\varphi}_{i_{j} i_{j+1}}\left(\inf _{B_{\alpha_{j+1}}} u_{i_{j+1}}\right) \leqslant \inf _{B_{\alpha_{j}}} u_{i_{j}}
$$

and the second estimate in Lemma 7.2 follows by iterating (91) $r$ times (note that $r<n(n-1)$ ), in view of the definition of $\tilde{\varphi}$ (see (72)).

By using the cooperativity assumption (H3) and the Lipschitz continuity of $g_{k}$ at $u=0$ (recall that $g(0, x)=0$ ), we have

$$
\mathcal{L}^{-}\left(D^{2} u_{k}, D u_{k}\right)-v u_{k} \leqslant f \quad \text { in } B_{2}
$$

for $k=1, \ldots, n$. The weak Harnack inequality for scalar equations (Proposition 4.4) yields

$$
\left|u_{k}\right|_{p_{k}, 2} \leqslant C_{\#}\left(\inf _{B_{2}} u_{k}+\|f\|_{L^{N}\left(B_{3}\right)}\right),
$$

where $p_{k}$ and $C_{\#}$ are positive constants which depend only on $N, \alpha_{0}, \gamma$, and $\nu$. We set $p=\min \left\{p_{1}, \ldots, p_{n}\right\}$ and note that $|\cdot|_{p, R}$ is non-decreasing in $p>0$. 
Next, we replace everywhere in the above proof each function $\varphi_{i j}$ for which $\varphi_{i j}(t)>0$ if $t>0$ by a Lipschitz continuous function $\hat{\varphi}_{i j}$ such that $\hat{\varphi}_{i j}(t) \leqslant \varphi_{i j}(t)$ and $\hat{\varphi}_{i j}(t)$ is strictly increasing, for $t \geqslant 0$ (if $\varphi_{i j}$ itself does not have these properties it is easy to see that such a function $\widehat{\varphi}_{i j}$ can be constructed). Of course, estimates (73) and (74) in Lemma 7.2 continue to hold.

We set

$$
\phi_{i j}(t)=\hat{\varphi}_{i j}^{-1}\left(\frac{t}{\kappa_{i j}}+\frac{C_{*}}{\kappa_{i j}}\|f\|_{L^{N}\left(B_{3}\right)}\right)
$$

and

$$
\Phi_{0}\left(t,\|f\|_{L^{N}\left(B_{3}\right)}\right)=\sup _{k \neq l} \tilde{\phi}_{i_{r-1} l} \circ \tilde{\phi}_{i_{r-1} i_{r-2}} \circ \cdots \circ \tilde{\phi}_{i_{1} i_{2}}(t) \circ \tilde{\phi}_{k i_{1}}(t),
$$

where for each $(k, l), k \neq l$, the chain $\left\{i_{j}\right\}_{j=0}^{r}$ is chosen as in Lemma 7.1. Note that $\Phi_{0}(t, s)$ is continuous and increasing on $[0, \infty)^{2}$, and $\Phi_{0}(0,0)=0$.

Then estimate (74) can be recast in the form

$$
\inf _{B_{2}} u_{l} \leqslant \Phi_{0}\left(\inf _{B_{1}} u_{k},\|f\|_{L^{N}\left(B_{3}\right)}\right),
$$

for all $k, l=1, \ldots, n, k \neq l$.

Finally

$$
\begin{aligned}
\left|u_{1} \vee \cdots \vee u_{n}\right|_{p, 2} & \leqslant\left|u_{1}+\cdots+u_{n}\right|_{p, 2} \\
& \leqslant\left(n^{1-1 / p} \vee 1\right) \sum_{i=1}^{n}\left|u_{i}\right|_{p, 2} \\
& \leqslant\left(n^{1-1 / p} \vee 1\right) C_{\#}\left(\sum_{i=1}^{n} \inf _{B_{2}} u_{i}+n\|f\|_{L^{N}\left(B_{3}\right)}\right) \\
& \leqslant\left(n^{2-1 / p} \vee n\right) C_{\#} \Phi_{0}\left(\min _{1 \leqslant i \leqslant n} \inf _{B_{1}} u_{i},\|f\|_{L^{N}\left(B_{3}\right)}\right)+C\|f\|_{L^{N}} \\
& =C \Phi_{0}\left(\inf _{B_{1}} u_{1} \wedge \cdots \wedge u_{n},\|f\|_{L^{N}\left(B_{3}\right)}\right)+C\|f\|_{L^{N}\left(B_{3}\right)} \\
& =: \Phi\left(\inf _{B_{1}} u_{1} \wedge \cdots \wedge u_{n},\|f\|_{L^{N}\left(B_{3}\right)}\right),
\end{aligned}
$$

which concludes the proof of Proposition 3.2.

The full Harnack inequality is an immediate consequence of Propositions 3.1 and 3.2.

\section{Part II. Linear elliptic systems}

\section{The estimates in the linear case}

In this section we restate and extend our results from Section 3 in the setting of linear weakly coupled cooperative elliptic systems of second order. In this more simple but important case we obtain more precise and easier-to-state results. In Section 10 we discuss some extensions and give some open problems and counterexamples. 
We study the system

$$
\left\{\begin{aligned}
L_{1} u_{1}+c_{11}(x) u_{1}+c_{12}(x) u_{2}+\cdots+c_{1 n}(x) u_{n} & =f_{1}(x), \\
L_{2} u_{2}+c_{21}(x) u_{1}+c_{22}(x) u_{2}+\cdots+c_{2 n}(x) u_{n} & =f_{2}(x), \\
& \vdots \\
L_{n} u_{n}+c_{n 1}(x) u_{1}+c_{n 2}(x) u_{2}+\cdots+c_{n n}(x) u_{n} & =f_{n}(x)
\end{aligned}\right.
$$

in the bounded domain $\Omega \subset \mathbb{R}^{N} ; n, N \geqslant 1$.

In order to simplify the notations we write (95) in the form

$$
L u+\mathcal{C} u=\vec{f},
$$

where $L=\operatorname{diag}\left(L_{1}, \ldots, L_{n}\right), \mathcal{C}(x)=\left(c_{i j}(x)\right)_{i, j=1}^{n}, u=\left(u_{1}, \ldots, u_{n}\right)^{\mathrm{T}}$, and $\vec{f}=\left(f_{1}, \ldots, f_{n}\right)^{\mathrm{T}}$.

The second-order elliptic operators $L_{1}, \ldots, L_{n}$ are supposed to be in general non-divergence form

$$
L_{k}=\sum_{i, j=1}^{N} a_{i j}^{k}(x) \frac{\partial^{2}}{\partial x_{i} \partial x_{j}}+\sum_{i=1}^{N} b_{i}^{k}(x) \frac{\partial}{\partial x_{i}},
$$

and to be uniformly elliptic:

(L1) there exists $\alpha_{0} \in(0,1)$ such that for all $\xi \in \mathbb{R}^{N}$, all $k=1, \ldots, n$, and almost every $x \in \Omega$ we have

$$
\alpha_{0}|\xi|^{2} \leqslant \sum_{i, j=1}^{N} a_{i j}^{k}(x) \xi_{i} \xi_{j} \leqslant \alpha_{0}^{-1}|\xi|^{2}
$$

We assume that the operators $L_{1}, \ldots, L_{n}$ and the matrix $\mathcal{C}$ have bounded measurable coefficients, with

$$
\max _{1 \leqslant k \leqslant n} \sum_{i=1}^{N}\left\|b_{i}^{k}\right\|_{L^{\infty}(\Omega)}^{2}=b^{2} \leqslant v, \quad \max _{1 \leqslant k \leqslant n} \sum_{i=1}^{n}\left\|c_{k i}\right\|_{L^{\infty}(\Omega)} \leqslant v .
$$

We assume that the system (95) is cooperative, that is, for all indices $i, j \in\{1, \ldots, n\}$, with $i \neq j$,

(L3) $c_{i j} \geqslant 0$ a.e. in $\Omega$.

Finally, we assume

(L4) $\vec{f} \in L^{N}\left(\Omega, \mathbb{R}^{n}\right)$,

and consider $L^{N}$-viscosity solutions $u \in C\left(\Omega, \mathbb{R}^{n}\right)$ of (95). Recall that any $u \in W_{\text {loc }}^{2, N}\left(\Omega, \mathbb{R}^{n}\right)$ which satisfies (95) a.e. in $\Omega$ is a viscosity solution.

The first result is the ABP estimate for (95). We prove it under an assumption which is milder that (H5) and (H6). Actually, assumption $\Psi$ below is sharp, in a sense which will become clear later (see Section 14).

Theorem 8.1 (ABP estimate). (a) We suppose that

( $\Psi)$ there exists a function $\Psi=\left(\psi_{1}, \ldots, \psi_{n}\right) \in W_{\text {loc }}^{2, p}\left(\Omega, \mathbb{R}^{n}\right) \cap C\left(\bar{\Omega}, \mathbb{R}^{n}\right)$, for some $p>N$, such that

$$
\begin{cases}L \Psi+\mathcal{C} \Psi \leqslant 0 & \text { a.e. in } \Omega \\ \Psi>0 & \text { in } \bar{\Omega} .\end{cases}
$$


If (L1)-(L4) hold and $u \in C\left(\bar{\Omega}, \mathbb{R}^{n}\right)$ satisfies

$$
L u+\mathcal{C} u \geqslant-\vec{f} \quad \text { in } \Omega,
$$

then

$$
\sup _{\Omega}\left(u_{1} \vee \cdots \vee u_{n}\right) \leqslant C\left(\sup _{\partial \Omega}\left(u_{1}^{+} \vee \cdots \vee u_{n}^{+}\right)+\left\|f^{+}\right\|_{L^{N}(\Omega)}\right)
$$

(recall $f=f_{1} \vee \cdots \vee f_{n}$ ). Respectively, if $u$ is such that $L u+\mathcal{C} u \leqslant \vec{f}$ then

$$
-\inf _{\Omega}\left(u_{1} \wedge \cdots \wedge u_{n}\right) \leqslant C\left(\sup _{\partial \Omega}\left(u_{1}^{-} \vee \cdots \vee u_{n}^{-}\right)+\left\|f^{+}\right\|_{L^{N}(\Omega)}\right) .
$$

The constant $C$ depends on $N, \alpha_{0}, v, \Psi$, and $\operatorname{diam} \Omega$.

(b) If instead of $(\Psi)$ one assumes in (a) the stronger (take $\Psi=(1, \ldots, 1))$ condition

$$
\sum_{j=1}^{n} c_{i j}(x) \leqslant 0 \text { a.e. in } \Omega, \text { for every } i \in\{1, \ldots, n\},
$$

then the following stronger conclusion holds true

$$
\sup _{\Omega} u_{1} \vee \cdots \vee u_{n} \leqslant \sup _{\partial \Omega} u_{1}^{+} \vee \cdots \vee u_{n}^{+}+C_{\mathrm{ABP}}\left\|f^{+}\right\|_{L^{N}(\Omega)},
$$

and, respectively,

$$
-\inf _{\Omega}\left(u_{1} \wedge \cdots \wedge u_{n}\right) \leqslant \sup _{\partial \Omega}\left(u_{1}^{-} \vee \cdots \vee u_{n}^{-}\right)+C_{\mathrm{ABP}}\left\|f^{+}\right\|_{L^{N}(\Omega)},
$$

where the constant $C_{\mathrm{ABP}}$ depends only on $N, \alpha_{0}, v$, and $\operatorname{diam} \Omega$.

Remark 1. Taking $n=1, u \in W_{\mathrm{loc}}^{2, N}(\Omega, \mathbb{R})$ in (100), we obtain Theorem 9.1 in [22] (compare also with [2], Theorem 1.3). This means our results contain the classical ABP estimate for scalar equations.

Remark 2. Hypothesis $(\Psi)$ implies that the matrix operator $L+\mathcal{C}$ satisfies the maximum principle in $\Omega$ (see [16] and Section 14 of our paper).

Remark 3. In the case when the second order coefficients of the elliptic operators $L_{1}, \ldots, L_{n}$ are continuous functions and $\partial \Omega$ has some regularity, we can weaken hypothesis $(\Psi)$. More precisely, instead of $\Psi>0$ in $\bar{\Omega}$ we could suppose that $\Psi>0$ in $\Omega$, with either $\Psi \not \equiv 0$ on $\partial \Omega$ or $L \Psi+\mathcal{C} \Psi \not \equiv 0$ in $\Omega$. This weaker condition will be shown to be equivalent to $(\Psi)$ (see in particular Lemma 14.1 in Section 14).

Remark 4. The dependence in $\Psi$ of the constant in Theorem 8.1 is expressed in terms of upper bounds on

$$
\left|\frac{\nabla \Psi}{\psi_{1} \wedge \cdots \wedge \psi_{n}}\right|_{L^{\infty}(\Omega)} \quad \text { and } \quad\left|\frac{\psi_{1} \vee \cdots \vee \psi_{n}}{\psi_{1} \wedge \cdots \wedge \psi_{n}}\right|_{L^{\infty}(\Omega)} \text {. }
$$

We turn to the Harnack inequality for non-negative solutions of (95).

Our first goal is to describe precisely the way system (95) can force the functions $u_{1}, \ldots, u_{n}$ to depend on each other.

Let us restate the definition of a fully coupled system in the linear case.

Definition 8.1. A matrix $\mathcal{C}(x)=\left(c_{i j}(x)\right)_{i, j=1}^{n}$, which satisfies (H3), is called irreducible in $\Omega$, and the system $L u+\mathcal{C} u=f$ is called fully coupled in $\Omega$, provided for any non-empty sets $I, J \subset\{1, \ldots, n\}$ such that $I \cap J=\emptyset$ and $I \cup J=\{1, \ldots, n\}$, there exist $i_{0} \in I$ and $j_{0} \in J$ for which

$$
\text { meas }\left\{x \in \Omega \mid c_{i_{0} j_{0}}(x)>0\right\}>0 .
$$


For simplicity, when (102) holds we write $c_{i_{0} j_{0}} \not \equiv 0$ in $\Omega$.

Next we give a notion of partial coupling for a non-fully coupled system. It is easy to see, by renumbering lines and columns (i.e., by permuting the indices of $u_{1}, \ldots, u_{n}$ ), that any matrix $\mathcal{C}$ can be written in the block triangular form

$$
\mathcal{C}=\left(\mathcal{C}_{k l}\right)_{k, l=1}^{m},
$$

so that the matrix which we obtain still satisfies (H3); here $1 \leqslant m \leqslant n, \mathcal{C}_{k l}$ are $t_{k} \times t_{l}$ matrices, $\sum_{k=1}^{m} t_{k}=n, \mathcal{C}_{k k}$ is an irreducible matrix for all $k=1, \ldots, m$, and $\mathcal{C}_{k l} \equiv 0$ in $\Omega$, for all $k, l \in\{1, \ldots, m\}$ with $k<l$. Note that $m=1$ means $\mathcal{C}$ is irreducible, while $m=n$ means $\mathcal{C}$ is in triangular form.

From now on, we suppose that $\mathcal{C}(x)$ is written in the form (103). We set $s_{0}=0, s_{k}=\sum_{i=1}^{k} t_{i}, S_{k}=$ $\left\{s_{k-1}+1, \ldots, s_{k}\right\}$ and

$$
\overline{u_{k}}=u_{s_{k-1}+1} \vee \cdots \vee u_{s_{k}}, \quad \underline{u_{k}}=u_{s_{k-1}+1} \wedge \cdots \wedge u_{s_{k}},
$$

for all $k \in\{1, \ldots, m\}$.

Definition 8.2. Let (95) be a non-fully coupled system and let $k>l$, for some $k, l \in\{1, \ldots, m\}$. We call system (95) ( $k l$ )-partially coupled, provided there exist indices $(i, j) \in S_{k} \times S_{l}$ such that $c_{i j} \not \equiv 0$ in $\Omega$. For simplicity, in this case we write $\mathcal{C}_{k l} \not \equiv 0$ in $\Omega$.

We fix a point $x_{0} \in \Omega$ and a ball $B_{3 R}:=B\left(x_{0}, 3 R\right) \subset \Omega$. We suppose the matrix $\mathcal{C}(x)$ is written in the form (103) in $B_{3 R}$ (i.e., $\mathcal{C}_{k l} \equiv 0$ in $B_{3 R}$ for $k<l$ and $\mathcal{C}_{k k}$ are irreducible in $B_{3 R}$ ).

We set, for all $k, l \in\{1, \ldots, m\}$,

$$
\Lambda_{k l}\left(B_{3 R}\right)=\left\{(i, j) \mid(i, j) \in S_{k} \times S_{l}, i \neq j \text { and } c_{i j} \not \equiv 0 \text { in } B_{3 R}\right\}, \quad \Lambda=\bigcup_{k, l=1}^{m} \Lambda_{k l}
$$

( $\Lambda$ can be empty, if (95) is totally decoupled) and fix $\alpha \in(0,3)$ such that $\Lambda_{k l}\left(B_{\alpha R}\right)=\Lambda_{k l}\left(B_{3 R}\right)$, for all $k, l \in\{1, \ldots, m\}$.

We shall prove a Harnack inequality in the ball $B_{\alpha R}$. To avoid heavy notations, we take $\alpha=1$ (in the general case the constants in the Harnack inequality depend on $3-\alpha)$. We set, for all $(i, j) \in \Lambda$,

$$
\omega_{i j}=\left\{x \in B_{R} \mid c_{i j}(x) \geqslant \rho\right\},
$$

where $\rho>0$ is taken so that meas $\left(\omega_{i j}\right)>0$.

Theorem 8.2 (Harnack inequality). Suppose (L1) through (L4) are satisfied and let $u \geqslant 0$ be a solution of (95) in $B_{3 R}$. Then

$$
\sup _{B_{R}} \overline{u_{k}} \leqslant C\left(\inf _{B_{R}} \underline{u_{k}}+R\|f\|_{L^{N}\left(B_{3 R}\right)}\right),
$$

for all $k \in\{1, \ldots, m\}$.

If, in addition, (95) is (kl)-partially coupled, then

$$
\sup _{B_{R}} \overline{u_{k}} \vee \overline{u_{l}} \leqslant C\left(\inf _{B_{R}} \underline{u_{k}}+R\|f\|_{L^{N}\left(B_{3 R}\right)}\right) .
$$

The constants $C$ in (105) and (106) depend only on $n, N, \alpha_{0}, \nu R^{2}, \rho R^{2}$, and a positive lower bound for $R^{-N}$ meas $\left(\omega_{i j}\right),(i, j) \in \Lambda \cap\left\{1, \ldots, s_{k}\right\}^{2}$. 
Counterexample. We recall that our results fail for general non-cooperative systems. For example, the functions $u=|x|^{2}, v=2 N$ solve the system

$$
\left\{\begin{array}{l}
\Delta u-v=0, \quad \text { in } B_{1} \subset \mathbb{R}^{N}, \\
\Delta v=0
\end{array}\right.
$$

and violate (106). See also the counterexamples at the end of Section 3.

A particular case of (105) is the following

Corollary 8.1. Under the hypotheses of Theorem 8.2 we have

$$
\sup _{B_{R}} u_{i} \leqslant C\left(\inf _{B_{R}} u_{i}+R\|f\|_{L^{N}\left(B_{3 R}\right)}\right)
$$

for all $i=1, \ldots, n$.

If, in addition, system (95) is fully coupled, then

$$
\sup _{B_{R}} u_{1} \vee \cdots \vee u_{n} \leqslant C\left(\inf _{B_{R}} u_{1} \wedge \cdots \wedge u_{n}+R\|f\|_{L^{N}\left(B_{3 R}\right)}\right) .
$$

Let us recall the earlier results on Harnack inequalities for elliptic systems. Extending results by Mandras [33], Chen and Zhao [10] obtained Corollary 8.1 for strong solutions of (95), in the case $\vec{f} \equiv 0$, under Hölder regularity assumptions on the coefficients of the elliptic operators $L_{1}, \ldots, L_{n}$. Their proof makes use of estimates on the Green functions of $L_{1}, \ldots, L_{n}$. The result of Chen and Zhao was shown to hold for $a_{i j}^{k} \in C^{0,1}, b_{i}^{k} \in L^{\infty}$ by Arapostatis, Ghosh and Marcus [1]. All these works relied on typically "linear" tools which require $\vec{f} \equiv 0$ and lead to the additional regularity assumptions on the coefficients of the elliptic operators. We note that, using a NashMoser type iteration technique, Muscalu [35] recently obtained a weak form of the Harnack inequality for a class of elliptic systems in divergence form.

Finally, we state the two half-Harnack inequalities.

Proposition 8.1 (local maximum principle). Suppose hypotheses (L1) through (L4) hold. Let

$$
L u+\mathcal{C} u \geqslant-\vec{f}
$$

in $B_{3 R}$. Then for all $p>0$ we have

$$
\sup _{B_{R}} u_{1} \vee \cdots \vee u_{n} \leqslant C\left(\left|u_{1}^{+} \vee \cdots \vee u_{n}^{+}\right|_{p, 2 R}+R\left\|f_{1}^{+} \vee \cdots \vee f_{n}^{+}\right\|_{L^{N}\left(B_{2 R}\right)}\right),
$$

where $C=C\left(N, \alpha_{0}, \nu R^{2}, p\right)$.

Proposition 8.2 (weak Harnack inequality). Suppose hypotheses (L1) through (L4) hold and let $u \geqslant 0$ satisfy

$$
L u+\mathcal{C} u \leqslant \vec{f}
$$

in $B_{3 R}$. Then there exists a number $p=p\left(N, n, \alpha_{0}, v R^{2}\right)>0$ such that for any $k \in\{1, \ldots, m\}$,

$$
\left|\overline{u_{k}}\right|_{p, 2 R} \leqslant C\left(\inf _{B_{R}} u_{k}+R\left\|f_{s_{k-1}+1}^{+} \vee \cdots \vee f_{s_{k}}^{+}\right\|_{L^{N}\left(B_{3 R}\right)}\right),
$$

and, in case (95) is ( $k l)$-partially coupled,

$$
\left|\overline{u_{k}} \vee \overline{u_{l}}\right|_{p, 2 R} \leqslant C\left(\inf _{B_{R}} \underline{u_{k}}+R\left\|f_{1}^{+} \vee \cdots \vee f_{k}^{+}\right\|_{L^{N}\left(B_{3 R}\right)}\right),
$$

where $C$ is as in Theorem 8.2. 


\section{Proofs}

We begin with the proof of the linear ABP estimate (Theorem 8.1). First, the statement in (b) is a particular case of Theorem 3.1 in Section 3.

In case system (95) satisfies condition $(\Psi)$, we make the following change of functions

$$
\bar{u}_{k}=\frac{u_{k}}{\psi_{k}}, \quad \bar{f}_{k}=\frac{f_{k}}{\psi_{k}} .
$$

A simple computation yields

$$
\frac{1}{\psi_{k}} L_{k} u_{k}=\bar{L}_{k} \bar{u}_{k}+\bar{u}_{k}\left(\frac{L_{k} \psi_{k}}{\psi_{k}}\right)
$$

for all $k=1, \ldots, n$, where, as in Lemma 4.2,

$$
\bar{L}_{k}=L_{k}+2 \sum_{i, j=1}^{N} a_{i j}^{k}(x) \frac{\partial_{i} \psi_{k}(x)}{\psi_{k}(x)} \frac{\partial}{\partial x_{j}} .
$$

We have then, by Lemma 4.2(b),

$$
\bar{L}_{k} \bar{u}_{k}+\sum_{j=1}^{n} \bar{c}_{k j} \bar{u}_{j} \geqslant-\bar{f}_{k},
$$

where

$$
\bar{c}_{k j}(x)=\frac{1}{\psi_{k}}\left(c_{k j} \psi^{j}+\delta_{k j} L_{k} \psi_{k}\right) .
$$

We see that $(\Psi)$ implies

$$
\sum_{j=1}^{n} \bar{c}_{k j}(x) \leqslant 0 \quad \text { in } \Omega,
$$

for all $k=1, \ldots, n$. Thus, by making the change of functions (113), we obtain a new cooperative system which satisfies (99). By applying the ABP estimate for such systems, which we already have, we obtain

$$
\sup _{\Omega} u_{1} \vee \cdots \vee u_{n} \leqslant \frac{\sup _{\Omega} \psi_{1} \vee \cdots \vee \psi_{n}}{\inf _{\Omega} \psi_{1} \wedge \cdots \wedge \psi_{n}}\left(\sup _{\partial \Omega} u_{1}^{+} \vee \cdots \vee u_{n}^{+}+C\left\|f^{+}\right\|_{L^{N}(\Omega)}\right) \text {. }
$$

Theorem 8.1 is proved.

The linear local maximum principle (Proposition 8.1) is a consequence of Proposition 3.1.

The proofs of the Harnack and the weak Harnack inequalities will be carried out through an induction argument. We use induction with respect to $m$, where, we recall, $m$ is the number of irreducible blocks which appear when we write the matrix $\mathcal{C}$ in the form (103).

The case $m=1$ (that is, $\mathcal{C}$ is irreducible) is a consequence of the nonlinear Harnack inequality we already proved in Part I. Note that in the case of a linear system the functions $\varphi_{i j}$ are linear in $t$

$$
\varphi_{i j}=t\left(\inf _{x \in \omega_{i j}} \operatorname{ess} c_{i j}(x)\right) \geqslant \rho t, \quad(i, j) \in \Lambda,
$$

so that the basic estimate (74) reduces to

$$
\inf _{B_{1}} u_{i} \geqslant \kappa_{i j} \inf _{B_{2}} u_{j}-C_{*}\|f\|_{L^{N}\left(B_{3}\right)}, \quad i \neq j,
$$


and the function $\Phi_{0}$ which appears at the end of the proof of Theorem 3.2 is linear, $\Phi_{0}(t, s)=C_{1} t+C_{2} s$. Let us suppose Theorem 8.2 and Proposition 8.2 hold for systems with at most $m-1$ irreducible blocks, and let us have a system with $m$ irreducible blocks.

Note that, by the induction hypothesis, Theorem 8.2 and Proposition 8.2 hold for the system formed by the first $s_{m-1}$ equations in (95). It remains to prove (105), (106), (111) and (112) for $k=m$.

The inequality (111) is obtained by repeating the last argument in Section 7 and by using the fact that (117) holds for $i, j \in S_{m}, i \neq j$. Note that (L3) implies

$$
\left(L_{i}-c_{i i}^{-}\right) u_{i} \leqslant f_{i} \quad \text { in } B_{2}, i=1, \ldots, n,
$$

so that the weak Harnack inequality for scalar equations yields

$$
\left|u_{i}\right|_{p_{i}, 2} \leqslant C\left(\inf _{B_{2}} u_{i}+\left\|f^{+}\right\|_{L^{N}\left(B_{3}\right)}\right) .
$$

Let us prove (112). Fix $l \in\{1, \ldots, m-1\}$ such that $\mathcal{C}_{m l} \not \equiv 0$ in $B_{1}$. Let for example $c_{i_{0} j_{0}} \not \equiv 0$ in $B_{1}$, for some $i_{0} \in S_{m}, j_{0} \in S_{l}$. Then, by (117),

$$
\inf _{B_{3} / 2} u_{j_{0}} \leqslant C\left(\inf _{B_{2}} u_{i_{0}}+\left\|f_{i_{0}}^{+}\right\|_{L^{N}\left(B_{3}\right)}\right) .
$$

Using (111), (117), (118), (119) and the induction hypothesis, we obtain

$$
\begin{aligned}
\left|\overline{u_{m}} \vee \overline{u_{l}}\right|_{p, 2} & \leqslant C\left(\left|\overline{u_{m}}\right|_{p, 2}+\left|\overline{u_{l}}\right|_{p, 2}\right) \\
& \leqslant C\left(\sum_{i \in S_{m}} \inf _{B_{2}} u_{i}+\min _{j \in S_{l} B_{3 / 2}} u_{j}+\left\|f^{+}\right\|_{L^{N}\left(B_{3}\right)}\right) \\
& \leqslant C\left(\sum_{i \in S_{m}} \inf _{B_{2}} u_{i}+\inf _{B_{3 / 2}} u_{j_{0}}+\left\|f^{+}\right\|_{L^{N}\left(B_{3}\right)}\right) \\
& \leqslant C\left(2 \sum_{i \in S_{m}} \inf _{B_{2}} u_{i}+\left\|f^{+}\right\|_{L^{N}\left(B_{3}\right)}\right) \\
& \leqslant C\left(\min _{i \in S_{m} \inf _{1}} u_{i}+\left\|f^{+}\right\|_{L^{N}\left(B_{3}\right)}\right) \\
& =C\left(\inf _{B_{1}} u_{m}+\left\|f^{+}\right\|_{L^{N}\left(B_{3}\right)}\right),
\end{aligned}
$$

which proves (112).

Finally, let us prove (105) and (106). We distinguish two cases.

Case 1 . There exists a number $l \in\{1, \ldots, m-1\}$ such that $\mathcal{C}_{k l} \equiv 0$ in $B_{1}$, for all $k>l$.

In this case we remove from (95) the equations with numbers in $S_{l}$ and obtain a system to which the induction hypothesis applies.

Case 2. For all $l \in\{1, \ldots, m-1\}$ there exists $k>l$ such that $\mathcal{C}_{k l} \not \equiv 0$ in $B_{1}$.

In this case we can even prove that

$$
\sup _{B_{1}} u_{1} \vee \cdots \vee u_{n} \leqslant C\left(\inf _{B_{1}} \underline{u_{m}}+\|f\|_{L^{N}\left(B_{3}\right)}\right) .
$$

In view of Proposition 8.1 it suffices to prove that

$$
\left|\overline{u_{l}}\right|_{p, 2} \leqslant C\left(\inf _{B_{1}} \underline{u_{m}}+\|f\|_{L^{N}\left(B_{3}\right)}\right),
$$


for all $l \in\{1, \ldots, m\}$. For $l=m$ this follows from (111). On the other hand, the assumption of Case 2 implies, by (112), that for all $l \in\{1, \ldots, m-1\}$ there exists $k>l$ such that

$$
\left|\overline{u_{l}}\right|_{p, 2} \leqslant C\left(\inf _{B_{1}} \underline{u_{k}}+\|f\|_{L^{N}\left(B_{3}\right)}\right) .
$$

Finally, we take a sequence $l=k_{0}<k_{1}<\cdots<k_{r}=m$ such that the latter inequality holds between $u_{k_{i}}$ and $u_{k_{i+1}}$ and at most $m-1$ nested balls between $B_{1}$ and $B_{2}$. Iterating the inequality between each two of them, we obtain (121).

\section{Further results and some open problems}

While the hypotheses under which we prove our Harnack inequality seem natural - and the result seems complete in view of what is known for scalar equations - a number of questions remain to be answered about the ABP inequality, and even about the maximum principle. Namely, (H5) and (H6) are not optimal. For instance, one could expect that Theorem 3.1 holds only under the hypothesis

$$
\mathcal{C}(u, x)=\left(\frac{\partial c_{i}}{\partial u_{j}}(u, x)\right)_{i, j=1}^{n} \quad \text { is negative semi-definite for a.e. }(u, x) .
$$

Indeed (122) is more general than (H5) and (H6) in view of the following lemma.

Lemma 10.1. Let $M=\left(m_{i j}\right) \in \mathcal{M}_{n}(\mathbb{R})$ be a cooperative matrix, i.e., $m_{i j} \geqslant 0$ for $i \neq j$. Suppose either that

$$
\sum_{j=1}^{n} m_{i j} \leqslant 0 \quad \text { for all } i=1, \ldots, n
$$

or that

$$
m_{i j} \leqslant \bar{m}_{i j}
$$

where $\bar{M}=\left(\bar{m}_{i j}\right)$ is a negative semi-definite matrix (i.e., $(\bar{M} \xi, \xi) \leqslant 0$ for all $\left.\xi \in \mathbb{R}^{n}\right)$. Then $M$ is negative semidefinite.

We give the elementary proof of Lemma 10.1 at the end of this section, for the sake of completeness.

Although Theorem 3.1 holds under (122) for some particular systems, this proves to be false in general. This section contains a discussion on these points.

The problem is quite delicate, even in the linear case. In particular, there turns out to be important differences between systems with divergence and non-divergence form operators, between systems with autonomous (i.e., constant in the linear case) or non-autonomous zero-order terms, and between systems with the same or different linear elliptic operators.

To avoid technical complications, in this section we consider only strong solutions, that is, $u \in W_{\text {loc }}^{2, N}\left(\Omega, \mathbb{R}^{n}\right) \cap$ $C\left(\bar{\Omega}, \mathbb{R}^{n}\right)$.

First, it follows from Theorem 3.1(b) that the system

$$
\mathcal{L}^{+}\left(D^{2} u_{i}, D u_{i}\right)+(\mathcal{C} u)_{i}=0, \quad i=1, \ldots, n,
$$

satisfies $\mathrm{ABP}$ (that is, (4) holds), if $\mathcal{C}$ is a constant negative semi-definite matrix.

Next, we recall that ABP remains true under (122) for linear systems with elliptic operators in divergence form.

Proposition 10.1. Suppose $u \in W^{2, N}\left(\Omega, \mathbb{R}^{n}\right)$ satisfies

$$
L u+\mathcal{C}(x) u \geqslant-\vec{f}
$$


in the regular domain $\Omega$, where $L_{k}, k=1, \ldots, n$, can be written in the form

$$
L_{k}=\sum_{i, j=1}^{N} \partial_{i}\left(a_{i j}^{k}(x) \partial_{j}\right)
$$

for some $a_{i j}^{k} \in C^{1}(\Omega)$, and suppose $\mathcal{C}(x)=\mathcal{C}(u, x)$ satisfies (122). Then

$$
\sup _{\Omega} u_{1} \vee \cdots \vee u_{n} \leqslant C\left(\sup _{\partial \Omega} u_{1}^{+} \vee \cdots \vee u_{n}^{+}+\left\|f^{+}\right\|_{L^{N}(\Omega)}\right) \text {. }
$$

Remark. The assumption that the coefficients of the elliptic operators are regular can of course be relaxed by considering the weak formulation of (125). We have made these hypothesis for simplicity, in order to remain in the non-divergence framework.

Proof of Proposition 10.1. As we show later, it suffices to prove that $L+\mathcal{C}$ satisfies the maximum principle (since this implies $(\Psi)$ from Theorem 8.1, see Section 14, Theorem 14.1). Let $u$ be such that

$$
\left\{\begin{array}{l}
L u+\mathcal{C} u \leqslant 0 \quad \text { in } \Omega \\
u \geqslant 0 \quad \text { on } \partial \Omega
\end{array}\right.
$$

We have to show that $u \geqslant 0$ in $\Omega$. We use a standard argument. We multiply the $i$-th equation by $u_{i}^{-}$and integrate over $\Omega$. We obtain

$$
-\int_{\Omega}\left(A^{i} D u_{i}, D u_{i}^{-}\right) \mathrm{d} x+\int_{\Omega} \sum_{j=1}^{n} c_{i j} u_{j} u_{i}^{-} \leqslant 0, \quad i=1, \ldots, n,
$$

where $A^{i}=\left(a_{r s}^{i}\right)_{r, s}$. Summing over $i$ we obtain (recall that $u=u^{+}-u^{-}$)

$$
\sum_{i=1}^{n} \int_{\Omega}\left(A^{i} D u_{i}^{-}, D u_{i}^{-}\right) \mathrm{d} x-\sum_{i, j=1}^{n} c_{i j} u_{j}^{-} u_{i}^{-} \leqslant 0 .
$$

Hence, by ellipticity and (122),

$$
\alpha_{0} \sum_{i=1}^{n} \int_{\Omega}\left|D u_{i}^{-}\right|^{2} \mathrm{~d} x \leqslant \int_{\Omega}\left(\mathcal{C}(x) u^{-}, u^{-}\right) \mathrm{d} x \leqslant 0
$$

which implies $u_{i}^{-} \equiv$ const $=0, i=1, \ldots, n$.

The next result shows that the ABP inequality remains true for strong solutions of linear systems in nondivergence form under assumption (122), provided all elliptic operators $L_{i}$ coincide.

Proposition 10.2. Suppose $\mathcal{C}(x)=\mathcal{C}(u, x)$ satisfies (122), u satisfies (125) and that $L_{1}=\cdots=L_{n}$ is a scalar second-order operator with bounded measurable coefficients in the form (96). Then (126) holds true.

Remark. We do have to restrict here to strong supersolutions; we suspect that this result extends to viscosity supersolutions, although we do not have a proof.

Proof. By dividing each function $u_{i}$ by a solution of $L \psi=-1$ in $\widetilde{\Omega}, \Omega \Subset \widetilde{\Omega}$, we can reduce to a modified system with negative definite zero-order matrix, see (47) and the computations thereafter. We keep the same notations for simplicity. Hence we can assume

$$
(\mathcal{C}(x) \xi, \xi) \leqslant-\alpha|\xi|^{2} \quad \text { for all } \xi \in \mathbb{R}^{n} \text {, a.e. } x \in \Omega,
$$

for some $\alpha>0$. 
As we explained above, in order to establish (126) it is enough to show that the maximum principle holds true for $L+\mathcal{C}$ in $\Omega$, i.e., that (127) implies $u_{i}^{-} \equiv 0$ in $\Omega$, for all $i$. First, note that by the cooperativity assumption (L3) the $i$-th equation in (127) yields

$$
L_{1} u_{i}+c_{i i} u_{i}^{+}-\sum_{j=1}^{n} c_{i j} u_{j}^{-} \leqslant 0 .
$$

Let us denote by $\phi_{\varepsilon}$ the convolution of the function $z \rightarrow \frac{1}{2}\left(z_{-}\right)^{2}$ with a standard smoothing kernel $\rho_{\varepsilon}$ (that is, $\left.\int_{\mathbb{R}} \rho_{\varepsilon}=1, \rho_{\varepsilon} \geqslant 0, \rho_{\varepsilon} \in C_{0}^{\infty}(\mathbb{R}), \operatorname{supp} \rho_{\varepsilon} \subset(-\varepsilon, \varepsilon)\right)$.

Note that $\phi_{\varepsilon}^{\prime}(z)=\rho_{\varepsilon} *\left(-z^{-}\right)$, so that $\left|-\phi_{\varepsilon}^{\prime}\left(u_{i}\right)-u_{i}^{-}\right| \leqslant \varepsilon$. By multiplying (129) by $-\phi_{\varepsilon}^{\prime}\left(u_{i}\right)(\geqslant 0)$ and by using (128) we get

$$
-\sum_{i=1}^{n} \phi_{\varepsilon}^{\prime}\left(u_{i}\right) L_{1} u_{i} \leqslant-\alpha \sum_{i}\left(u_{i}^{-}\right)^{2}+C \varepsilon,
$$

where $C$ depends only on $\|u\|_{L^{\infty}(\Omega)}$. Observe that for any $w \in W_{\text {loc }}^{2, n}$ and any convex $\phi \in C^{2}$ we have the following well-known Kato inequality

$$
L_{1}(\phi(w)) \geqslant \phi^{\prime}(w) L_{1} w .
$$

We assume for contradiction that $\sum_{i}\left(u_{i}^{-}\right)^{2}>0$ and apply (131), with $w=u_{i}, \phi=\phi_{\varepsilon}$, to (130). This yields

$$
-L_{1}\left(\sum_{i=1}^{n} \phi_{\varepsilon}\left(u_{i}\right)\right) \leqslant-\sum_{i=1}^{n} \phi_{\varepsilon}^{\prime}\left(u_{i}\right) L_{1} u_{i} \leqslant 0
$$

for all $\varepsilon>0$ small enough. By the scalar maximum principle applied to (132), noticing that $u_{i} \geqslant 0$ on $\partial \Omega$ implies $\phi_{\varepsilon}\left(u_{i}\right)=\mathrm{O}(\varepsilon)$ on $\partial \Omega$, we get

$$
\sum_{i=1}^{n} \phi_{\varepsilon}\left(u_{i}\right) \leqslant C \varepsilon
$$

in $\Omega$. Taking $\varepsilon \rightarrow 0$ we get $u_{i}^{-} \equiv 0$ in $\Omega$ for all $i$, a contradiction.

In the nonlinear case we are able to prove that system (1) satisfies the maximum principle under (122), provided it is autonomous. We do not know whether ABP holds in this situation.

Proposition 10.3. Suppose $u \in C\left(\bar{\Omega}, \mathbb{R}^{n}\right)$ satisfies

$$
\left\{\begin{array}{l}
\mathcal{L}^{-}\left(D^{2} u_{i}, D u_{i}\right)+c_{i}(u) \leqslant 0 \quad \text { in } \Omega, \\
u \geqslant 0 \quad \text { on } \partial \Omega \\
i=1, \ldots, n
\end{array}\right.
$$

in the viscosity sense. Assume the functions $c_{i}$ satisfy (H0), (H3) and

$$
(c(v), v)=\sum_{i=1}^{n} c_{i}(v) v_{i} \leqslant 0 \quad \text { for all } v \in \mathbb{R}^{n} .
$$

Then $u \geqslant 0$ in $\Omega$.

Remark. Condition (135) is weaker than (122). This is natural, since in Proposition 10.3 we aim at a maximum principle only. 
Proof of Proposition 10.3. Set $u_{i}=\bar{u}_{i} \psi$, where $\psi$ is a strong solution of the equation

$$
\left\{\begin{array}{l}
\mathcal{L}^{-}\left(D^{2} \psi, D \psi\right)=-1 \text { in } \widetilde{\Omega} \\
\psi=0 \text { on } \partial \widetilde{\Omega}
\end{array}\right.
$$

with $\Omega \Subset \widetilde{\Omega}$. Up to adding a constant to $\gamma$ we have

$$
\left\{\begin{array}{l}
\mathcal{L}^{-}\left(D^{2} \bar{u}_{i}, D \bar{u}_{i}\right)+\hat{c}_{i}(\bar{u}) \leqslant 0 \quad \text { in } \Omega \\
u \geqslant 0 \quad \text { on } \partial \Omega \\
i=1, \ldots, n
\end{array}\right.
$$

where $\hat{c}$ is defined by

$$
\hat{c}_{i}(\bar{u})=\frac{1}{\psi} c_{i}(\bar{u} \psi)-\varepsilon \bar{u}_{i}, \quad \varepsilon=\frac{1}{\sup _{\Omega} \psi}
$$

(see Lemma 4.3 and (48)). Now system (136) satisfies all hypotheses of Proposition 10.3, with a strict inequality in (135), for all $v \in \mathbb{R}^{n} \backslash\{0\}$. For simplicity we write $c$ instead of $\hat{c}$ and $u$ instead of $\bar{u}$.

By (H3) we have

$$
\mathcal{L}^{-}\left(D^{2} u_{i}, D u_{i}\right)+c_{i}\left(-u_{1}^{-}, \ldots,-u_{i-1}^{-}, u_{i},-u_{i+1}^{-}, \ldots,-u_{n}^{-}\right) \leqslant 0 \quad \text { in } \Omega
$$

for all $i$. Hence

$$
-\mathcal{L}^{+}\left(D^{2} u_{i}^{-}, D u_{i}^{-}\right)+c_{i}\left(-u^{-}\right) \leqslant 0,
$$

since $u_{i}^{-}=-\min \left(u_{i}, 0\right)$ and the minimum of supersolutions is a supersolution. Let $\bar{x}_{i} \in \Omega$ be a point of maximum of the nonnegative function $u_{i}^{-}$. Set $\overline{c_{i}}=-c_{i}\left(-u_{1}^{-}\left(\bar{x}_{1}\right), \ldots,-u_{n}^{-}\left(\bar{x}_{n}\right)\right)$. We claim that $\overline{c_{i}} \geqslant 0$ for all $i$. If not, there exists $j \in\{1, \ldots, n\}$ such that $\overline{c_{j}}<0$ and, by continuity,

$$
c_{j}\left(-u_{1}^{-}\left(x_{1}\right), \ldots,-u_{n}^{-}\left(x_{n}\right)\right)>\frac{\overline{c_{i}}}{2},
$$

for $\left(x_{1}, \ldots, x_{n}\right) \in \mathcal{O}$, where $\mathcal{O}$ is a neighbourhood of $\left(\bar{x}_{1}, \ldots, \bar{x}_{n}\right)$, such that $u_{j}^{-}$achieves its maximum in $\mathcal{O}$ at $\bar{x}_{j}$. We get

$$
\mathcal{L}^{-}\left(D^{2}\left(u_{j}^{-}\left(\bar{x}_{j}\right)-u_{j}^{-}\right), D\left(u_{j}^{-}\left(\bar{x}_{j}\right)-u_{j}^{-}\right)\right)=-\mathcal{L}^{+}\left(D^{2} u_{j}^{-}, D u_{j}^{-}\right) \leqslant-\frac{\overline{c_{i}}}{2}<0
$$

in $\mathcal{O}$. By the scalar strong maximum principle $u_{j}^{-} \equiv u_{j}^{-}\left(\bar{x}_{j}\right)$ in $\mathcal{O}$, which is a contradiction with the last inequality.

Set $y_{i}=u_{i}^{-}\left(\bar{x}_{i}\right) \geqslant 0$ and $y=\left(y_{1}, \ldots, y_{n}\right)$. Then, by $\overline{c_{i}} \geqslant 0$ and (135),

$$
0 \leqslant \sum_{i=1}^{n}\left(-y_{i}\right) c_{i}(-y)<0
$$

unless $y_{i}=0$ for all $i$.

Finally we show, through a counterexample, that condition (122) is not sufficient to ensure the validity of the maximum principle in the non-autonomous case, even for a linear system.

Set $I=(-3,3)$ and define the functions $a, d \in C^{\infty}(I)$ as follows

$$
a(x)=\left\{\begin{array}{rl}
-\varepsilon & \text { if } x \in[-3,-1], \\
-\frac{2}{\varepsilon} & \text { if } x \in[1,3],
\end{array} \quad d(x)=\left\{\begin{aligned}
-\frac{2}{\varepsilon} & \text { if } x \in[-3,-1], \\
-\varepsilon & \text { if } x \in[1,3]
\end{aligned}\right.\right.
$$

and such that $a(x) d(x) \equiv 2$ in $I$. 
Let $v \in C^{2}(\bar{I})$ be a positive function such that

$$
v(-3)=v(3)=0, \quad v^{\prime \prime}>0 \quad \text { in }[-3,1) \cup(2,3] \quad \text { and } \quad v^{\prime \prime}<0 \quad \text { in }(1,2) .
$$

Set $u(x)=v(-x)$. It is easy to see that if $\varepsilon$ and $c_{0}$ are small enough the following inequalities hold

$$
\begin{cases}\mathcal{M}^{+}\left(D^{2} u\right)+a(x) u+v \geqslant 0 & \text { in } I, \\ \mathcal{M}^{+}\left(D^{2} v\right)+u+d(x) v \geqslant 0 & \text { in } I, \\ u, v=0 & \text { on } \partial I .\end{cases}
$$

This system satisfies (122) but it does not satisfy the maximum principle, since $u, v \nless 0$ in $\Omega$. Note also that (137) can be written as a linear system, by using Lemma 4.1 .

Proof of Lemma 10.1. Set $a_{i j}=\frac{1}{2}\left(m_{i j}+m_{j i}\right), \bar{a}_{i j}=\frac{1}{2}\left(\bar{m}_{i j}+\bar{m}_{j i}\right)$, and $A=\left(a_{i j}\right), \bar{A}=\left(\bar{a}_{i j}\right)$. If (123) holds one gets by a trivial computation

$$
(M \xi, \xi)=(A \xi, \xi) \leqslant-\sum_{i<j} a_{i j}\left(\xi_{j}-\xi_{i}\right)^{2} \leqslant 0 .
$$

In case (124) is verified $\left(a_{i j} \leqslant \bar{a}_{i j}\right.$ and $(\bar{A} \xi, \xi) \leqslant 0$ for all $\left.\xi \in \mathbb{R}^{n}\right)$ we set $B_{\varepsilon}=\varepsilon I-A, \bar{B}_{\varepsilon}=\varepsilon I-\bar{A}=\left(\bar{b}_{i j}\right)$, so that $\bar{B}_{\varepsilon}$ is positive definite. It is clearly enough to show that $B_{\varepsilon}$ is positive definite under the additional assumption that $A$ and $\bar{A}$ differ only in one entry, say

$$
a_{i j}=\bar{a}_{i j} \quad \text { if }(i, j) \neq\left(i_{0}, j_{0}\right) \quad \text { and } \quad a_{i_{0} j_{0}}<\bar{a}_{i_{0} j_{0}} .
$$

The result then follows easily by taking a chain of matrices each two consecutive elements of which differ only in one entry, and by letting $\varepsilon \rightarrow 0$.

So suppose (138) and set $B(t)=(1-t) \bar{B}_{\varepsilon}+t B_{\varepsilon}=\left(b_{i j}(t)\right)$. Let, as before, $M^{k l}$ denotes the submatrix obtained from an arbitrary matrix $M$ by removing its $k$-th line and $l$-th column. By (138) $\operatorname{det} B^{i_{0} j}(t)=\operatorname{det} \bar{B}^{i_{0} j}(t)$, for any $j \in\{1, \ldots, n\}$. By Cramer's rule and Lemma 5.3 we get

$$
\begin{aligned}
\operatorname{det} B(t) & =\sum_{j \neq j_{0}}(-1)^{i_{0}+j} b_{i_{0} j}(t) \operatorname{det} B^{i_{0} j}(t)+(-1)^{i_{0}+j_{0}} b_{i_{0} j_{0}}(t) \operatorname{det} B^{i_{0} j_{0}}(t) \\
& \geqslant \sum_{j \neq j_{0}}(-1)^{i_{0}+j} \bar{b}_{i_{0} j}(t) \operatorname{det} \bar{B}^{i_{0} j}(t)+(-1)^{i_{0}+j_{0}} \bar{b}_{i_{0} j_{0}}(t) \operatorname{det} \bar{B}^{i_{0} j_{0}}(t) \\
& =\operatorname{det} \bar{B}_{\varepsilon}>0 .
\end{aligned}
$$

It follows, by continuity in $t$, that all eigenvalues of $B(1)=B_{\varepsilon}$ are positive, i.e., that $B_{\varepsilon}$ is positive definite.

\section{Part III. Applications}

In the third part of the paper we give several applications of the results obtained in Parts I and II. We prove a maximum principle in unbounded domains and a sharp strong maximum principle for cooperative systems. An important application is the existence of a principal eigenvalue and a principal eigenfunction of a fully coupled system. This result permits us to obtain a necessary and sufficient condition for a cooperative (not necessarily fully coupled) system to satisfy the maximum principle. Finally, we show how our results can be applied to give Harnack type estimates for a class of higher order elliptic equations, including the biharmonic and the polyharmonic equation. We show the existence of a principal eigenvalue and a principal eigenfunction for these equations, in a sense which seems to be new.

In order to simplify the presentation all these applications are given in the linear case although most results (maximum principles, higher order equations) readily extend to nonlinear equations. 


\section{A maximum principle in unbounded domains}

We say that the operator $L+\mathcal{C}$ satisfies the maximum principle in $\Omega$ provided for any $u \in C\left(\bar{\Omega}, \mathbb{R}^{n}\right)$,

$$
\left\{\begin{array}{l}
L u+\mathcal{C} u \leqslant 0 \quad \text { in } \Omega \\
u \geqslant 0 \quad \text { on } \partial \Omega
\end{array}\right.
$$

implies $u \geqslant 0$ in $\Omega$. When $\Omega$ is bounded, it is well-known that the assumptions of Theorem 8.1 (the ABP estimate) are sufficient to ensure that $L+\mathcal{C}$ satisfies the maximum principle in $\Omega$ (see [15]). This fact is a particular case of Theorem 8.1.

The ABP estimate can also be used to derive a maximum principle in unbounded domains. The next proposition contains a result of this kind. General results of the same type for scalar equations can be found in [6].

Proposition 11.1. Let $\Omega \subseteq \mathbb{R}^{N}$ be a domain (bounded or unbounded). Suppose (L1) through (L4) hold and

$$
\sum_{j=1}^{n} c_{i j}(x) \leqslant-\delta<0 \quad \text { a.e. in } \Omega
$$

for all $i=1, \ldots, n$, and some $\delta>0$. Then there exists $\varepsilon_{0}\left(\delta, N, \alpha_{0}, v\right)>0$ such that

$$
\begin{cases}L u+\mathcal{C} u \leqslant 0 & \text { in } \Omega, \\ u \geqslant 0 & \text { on } \partial \Omega, \text { if } \partial \Omega \neq \emptyset, \\ u_{1}^{-}(x) \vee \cdots \vee u_{n}^{-}(x) \leqslant C \mathrm{e}^{\varepsilon_{0}|x|} & \text { in } \Omega, \text { for some } C>0,\end{cases}
$$

implies $u \geqslant 0$ in $\Omega$.

Proof. We take $\beta>0$ such that $2 \sqrt{N} \alpha_{0}^{-1} \beta^{2}+v \sqrt{N} \beta<\delta$ and set $\varepsilon_{0}=\beta / 2$. We make the change of functions $\bar{u}_{i}=u_{i} / g$, with $g(x)=\cosh \beta x_{1} \cdots \cosh \beta x_{N}$. Then $\bar{u}=\left(\bar{u}_{1}, \ldots, \bar{u}_{n}\right)$ satisfies

$$
\bar{L} \bar{u}+\overline{\mathcal{C}} \bar{u} \leqslant 0 \quad \text { in } \Omega,
$$

where $\bar{L}=\left(\bar{L}_{1}, \ldots, \bar{L}_{n}\right)$,

$$
\bar{L}_{k}=L_{k}+2 \beta \sum_{1 \leqslant i, j \leqslant N} a_{i j}^{k}(x) \tanh \beta x_{i} \frac{\partial}{\partial x_{j}}
$$

and

$$
\overline{\mathcal{C}}=\mathcal{C}+\operatorname{diag}\left(\frac{L_{1} g}{g}, \ldots, \frac{L_{n} g}{g}\right) .
$$

More precisely, $\overline{\mathcal{C}}=\left(\bar{c}_{k j}\right)_{k, j=1}^{n}$, with

$$
\bar{c}_{k j}=c_{k j}+\delta_{k j}\left(\beta^{2} \sum_{\substack{1 \leqslant l, m \leqslant N \\ l \neq m}} a_{l m}^{k} \tanh \beta x_{l} \tanh \beta x_{m}+\beta^{2} \sum_{1 \leqslant l \leqslant N} a_{l l}^{k}+\beta \sum_{1 \leqslant l \leqslant N} b_{l}^{k} \tanh \beta x_{l}\right) .
$$

Note that, because of the choice of $\beta$ and $\varepsilon_{0}, \overline{\mathcal{C}}$ satisfies condition (99) and $\bar{u}^{-} \rightarrow 0$ as $|x| \rightarrow+\infty, x \in \Omega$. By applying ABP inequality (Theorem 8.1, (101)) to $\bar{u}$ in balls of increasing radii, we obtain the conclusion of Proposition 11.1. 


\section{A sharp strong maximum principle}

An immediate consequence of the weak Harnack inequality (Proposition 8.2) is the following strong maximum principle. We recall we suppose that the zero order matrix $\mathcal{C}$ is written in the form (103) in $\Omega$.

Proposition 12.1. Assume (L1) through (L4) hold. Suppose $u \in C\left(\bar{\Omega}, \mathbb{R}^{n}\right)$ satisfies

$$
\begin{cases}L u+\mathcal{C} u \leqslant 0 & \text { in } \Omega \\ u \geqslant 0 & \text { in } \Omega .\end{cases}
$$

Let $1 \leqslant k \leqslant m$ and suppose there exists a point $x_{0} \in \Omega$ and an index $i \in S_{k}$ such that $u_{i}\left(x_{0}\right)=0$. Then $u_{j} \equiv 0$ for all $j \in S_{k}$. If, in addition, $1 \leqslant k<l \leqslant m$ are such that there exists a sequence $\left\{i_{j}\right\}_{j=0}^{r}$ for which

$$
k=i_{0}>i_{1}>\cdots>i_{r}=l \text { and } \mathcal{C}_{i_{j} i_{j+1}} \not \equiv 0 \text { in } \Omega
$$

then $u_{j} \equiv 0$ in $\Omega$, for all $j \in \bigcup_{j=0}^{r} S_{i_{j}}$.

Remark 1. In the particular case when the system is fully coupled $(m=1)$, Proposition 12.1 reduces to the known strong maximum principle (see [40]), which states that $u_{i}\left(x_{0}\right)=0$ for some $x_{0} \in \Omega$ and some $i \in\{1, \ldots, n\}$ implies $u \equiv 0$ in $\Omega$.

Remark 2. The strong maximum principle given by Proposition 12.1 is sharp in the sense that if a sequence as in (141) does not exist then, clearly, the system does not relate the functions with indices in $S_{k}$ to the functions with indices in $S_{l}$.

\section{Existence of a principal eigenvalue for a fully coupled system}

Throughout this and the next section we suppose that (L1) through (L3) hold and, in addition,

$$
a_{i j}^{k} \in C(\Omega),
$$

for all $i, j \in\{1, \ldots, N\}, k \in\{1, \ldots, n\}$. All functions considered belong to $W_{\text {loc }}^{2, q}\left(\Omega, \mathbb{R}^{n}\right) \cap C\left(\bar{\Omega}, \mathbb{R}^{n}\right)$, for all $q<\infty$ (except otherwise stated), so that, in contrast to the rest of the paper, all equalities and inequalities hold almost everywhere.

For simplicity we suppose that $\Omega$ is regular (for example, $\Omega$ satisfies a uniform exterior cone condition). All our results can be extended to arbitrary domains, by using the arguments in [2].

We set

$$
\begin{aligned}
\lambda_{1} & =\lambda_{1}(L+\mathcal{C}) \\
& =\sup \left\{\lambda \in \mathbb{R} \mid \text { there exists } \Psi \in W_{\text {loc }}^{2, N}\left(\Omega, \mathbb{R}^{n}\right) \text { such that } \Psi>0 \text { and }(L+\mathcal{C}+\lambda I) \Psi \leqslant 0 \text { in } \Omega\right\} .
\end{aligned}
$$

Our main result in this section is the following theorem.

Theorem 13.1. Suppose that $\mathcal{C}$ is an irreducible matrix (see Definition 8.1). Then (a) there exists a function $\Phi_{1} \in W_{\mathrm{loc}}^{2, q}\left(\Omega, \mathbb{R}^{n}\right) \cap C\left(\bar{\Omega}, \mathbb{R}^{n}\right), \forall q<\infty$, such that

$$
\begin{cases}\left(L+\mathcal{C}+\lambda_{1} I\right) \Phi_{1}=0 & \text { in } \Omega, \\ \Phi_{1}>0 & \text { in } \Omega, \\ \Phi_{1}=0 & \text { on } \partial \Omega .\end{cases}
$$

(b) There are no eigenvalues of $-(L+\mathcal{C})$ in the interval $\left(-\infty, \lambda_{1}\right)$; the vector $\Phi_{1}$ spans $\operatorname{Ker}\left(L+\mathcal{C}+\lambda_{1} I\right)$ in $W_{\text {loc }}^{2, N}\left(\Omega, \mathbb{R}^{n}\right) \cap C\left(\bar{\Omega}, \mathbb{R}^{n}\right)$ under the Dirichlet boundary condition. 
(c) Assume there is a function $\Psi \in W_{\mathrm{loc}}^{2, N}\left(\Omega, \mathbb{R}^{n}\right)$ such that

$$
\Psi>0 \text { and }(L+\mathcal{C}) \Psi \leqslant 0 \text { in } \Omega .
$$

Then either $\lambda_{1}>0$ or $\lambda_{1}=0$ and $\Psi=$ const $\cdot \Phi_{1}$.

(d) If $\bar{\Psi} \in W_{\text {loc }}^{2, N}\left(\Omega, \mathbb{R}^{n}\right) \cap C\left(\bar{\Omega}, \mathbb{R}^{n}\right)$ satisfies

$$
\begin{cases}\left(L+\mathcal{C}+\lambda_{1} I\right) \bar{\Psi} \leqslant 0 & \text { in } \Omega \\ \bar{\Psi} \geqslant 0 & \text { on } \partial \Omega\end{cases}
$$

then $\bar{\Psi}=$ const $\cdot \Phi_{1}$.

(e) If we normalize $\Phi_{1}=\left(\phi_{1,1}, \ldots, \phi_{1, n}\right)$ in such a way that

$$
\min _{1 \leqslant i \leqslant n} \phi_{1, i}\left(x_{0}\right)=1
$$

for some $x_{0} \in \Omega$, then

$$
\sup _{\Omega} \Phi_{1}:=\sup _{\Omega} \phi_{1,1} \vee \cdots \vee \phi_{1, n} \leqslant C,
$$

where $C$ depends only on $x_{0}, \Omega$ and the same quantities as the constant which appears in the Harnack inequality (Theorem 8.2 in Part II).

Remark 1. Supposing a priori that $\lambda_{1}>0$ (he actually uses a hypothesis of type $(\Psi)$ and proves his hypothesis is equivalent to $\lambda_{1}>0$ ), Sweers proved parts (a) and (b) of Theorem 13.1 in [40] (his proof relies on the KreinRutman technique; see also [3] for the case of a non-regular domain). We are going to use this result in the proof of Theorem 13.1.

Remark 2. If the boundary of $\Omega$ is not regular the principal eigenfunction may not belong to $C(\bar{\Omega})$ and $\Phi_{1}=0$ on $\partial \Omega$ only in a certain sense (as in [2] and [3]).

Remark 3. In [2] Berestycki, Nirenberg and Varadhan made a deep study of the properties of the principal eigenvalue and the principal eigenfunction of a scalar elliptic operator in a general domain. The basic tools they used are the ABP and the Harnack-Krylov-Safonov inequalities for scalar equations. Since we now have such inequalities for cooperative elliptic systems, it is only a matter of technique to show that all results in [2] have their analogues for systems. Here and in the next section we present some of these analogues (and often merely adapt the proofs in [2] to the case of a system). To extend to systems the rest of the results in [2] is left to the interested reader.

Remark 4. The hypothesis that the system is fully coupled can be relaxed in Theorem 13.1.

Remark 5. In [25] Hess considered a related eigenvalue problem. He showed that the equation $L \Phi+\mu \mathcal{C} \Phi=0$ has a solution $(\mu, \Phi)$ under Dirichlet boundary conditions, with $\mu>0, \Phi>0$ in $\Omega$, provided $c_{k k}^{+} \not \equiv 0$ for some $k \in\{1, \ldots, n\}$. In other words, Hess gave a condition on $\mathcal{C}$ under which one can find a positive constant $\mu$ such that $\lambda_{1}(L+\mu \mathcal{C})=0$, in terms of our definition of $\lambda_{1}$.

Proof of Theorem 13.1. It follows from the definition of $\lambda_{1}$ that for any $\lambda<\lambda_{1}$ the matrix operator $L+\mathcal{C}+\lambda I$ satisfies the hypotheses of Theorem 1.1 in [40] (namely, this operator is cooperative, fully coupled, and satisfies a condition of type $(\Psi))$. This theorem implies the existence of couple $\left(\mu_{\lambda}, \Phi_{\lambda}\right)$ such that $\Phi_{\lambda} \in W_{\text {loc }}^{2, N}\left(\Omega, \mathbb{R}^{N}\right) \cap$ $C\left(\bar{\Omega}, \mathbb{R}^{N}\right), \mu_{\lambda}>0$ and

$$
\begin{cases}\left(L+\mathcal{C}+\left(\lambda+\mu_{\lambda}\right) I\right) \Phi_{\lambda}=0 & \text { in } \Omega, \\ \Phi_{\lambda}>0 & \text { in } \Omega, \\ \Phi_{\lambda}=0 & \text { on } \partial \Omega .\end{cases}
$$


We claim that

$$
\lambda<\lambda+\mu_{\lambda} \leqslant \lambda_{1} \leqslant \frac{C_{1}}{R^{2}},
$$

where $C_{1}=C_{1}\left(N, \alpha_{0}, v\right)$ and $R>0$ is such that $\Omega$ contains a ball of radius $R$.

The first inequality in (143) is obvious. The second inequality follows from the definition of $\lambda_{1}$ and (142). The last inequality in (143) is known to hold if $\lambda_{1}$ is replaced by $\lambda_{1}\left(L_{i}+c_{i i}\right)$ - the principal eigenvalue of the scalar operator $L_{i}+c_{i i}$ in $\Omega$, for any $i=1, \ldots, n$ (this is Lemma 1.1, page 51 in [2]). On the other hand, since $\mathcal{C}$ is cooperative,

$$
(L+\mathcal{C}+\lambda I) \Phi \leqslant 0 \quad \text { implies } \quad\left(L_{i}+c_{i i}+\lambda\right) \phi_{i} \leqslant 0
$$

$\left(\Phi=\left(\phi_{1}, \ldots, \phi_{n}\right)\right)$, hence $\lambda_{1} \leqslant \lambda_{1}\left(L_{i}+c_{i i}\right)$, for all $i=1, \ldots, n$.

We now fix $\delta=\delta\left(N, \alpha_{0}, \nu, \Omega\right)>0$ such that

$$
\delta<\left\{2 C_{\mathrm{ABP}}\left(v+\frac{C_{1}}{R^{2}}\right)\right\}^{-N}
$$

( $C_{\mathrm{ABP}}$ is the constant which appears in Theorem 8.1(b)).

Let $x_{0} \in \Omega$. We normalize the vector $\Phi_{\lambda}=\left(\phi_{\lambda, 1}, \ldots, \phi_{\lambda, n}\right)$ by dividing the equation in (142) by $\min _{1 \leqslant i \leqslant n} \phi_{\lambda, i}\left(x_{0}\right)>0$, so that we can suppose

$$
\phi_{\lambda, i}\left(x_{0}\right) \geqslant 1, \quad \text { for all } i=1, \ldots, n,
$$

and

$$
\phi_{\lambda, 1} \wedge \cdots \wedge \phi_{\lambda, n}\left(x_{0}\right)=1
$$

We take a compact set $K \subset \Omega$ such that we have $x_{0} \in K, B_{R} \subset K, \Lambda(K)=\Lambda(\Omega)(\Lambda$ is defined in Section 8 , page 38), and

$$
\operatorname{meas}(\Omega \backslash K)<\delta \text {. }
$$

It follows from (144) and our Harnack inequality (Corollary 8.1 in Part II) that

$$
\sup _{K} \phi_{\lambda, 1} \vee \cdots \vee \phi_{\lambda, n} \leqslant C_{2}
$$

(the constant $C$, indexed or not, depends only on the appropriate quantities, in particular, $C$ is independent of $\lambda$ ).

Set $e=(1,1, \ldots, 1)$ and $\bar{\Phi}_{\lambda}=\Phi_{\lambda}-C_{2} e$, so that

$$
\bar{\Phi}_{\lambda} \leqslant 0 \quad \text { on } \partial(\Omega \backslash K) \text {. }
$$

We have, by (142) and (143),

$$
\begin{aligned}
(L+\mathcal{C}-v I) \bar{\Phi}_{\lambda} & =-\left(v+\lambda+\mu_{\lambda}\right) \Phi_{\lambda}-C_{2}(\mathcal{C}-v I) e \\
& \geqslant-\left(v+\frac{C_{1}}{R^{2}}\right) \Phi_{\lambda} \quad \text { in } \Omega \backslash K,
\end{aligned}
$$

where we have used the fact that (L2) implies $(\mathcal{C}-v I) e \leqslant 0$. This fact also shows that the operator in the left-hand side of (147) satisfies the hypotheses of the ABP estimate (Theorem 8.1(b)). Applying this estimate to (147), we obtain

$$
\begin{aligned}
\sup _{\Omega \backslash K} \phi_{\lambda, 1} \vee \cdots \vee \phi_{\lambda, n}-C_{2} & \leqslant C_{\mathrm{ABP}}\left(v+\frac{C_{1}}{R^{2}}\right) \delta^{1 / N} \sup _{\Omega \backslash K} \phi_{\lambda, 1} \vee \cdots \vee \phi_{\lambda, n} \\
& \leqslant \frac{1}{2} \sup _{\Omega \backslash K} \phi_{\lambda, 1} \vee \cdots \vee \phi_{\lambda, n} .
\end{aligned}
$$


By combining (145) and (148) we obtain

$$
\left\|\Phi_{\lambda}\right\|_{L^{\infty}(\Omega)}=\sup _{\Omega} \phi_{\lambda, 1} \vee \cdots \vee \phi_{\lambda, n} \leqslant C_{3} .
$$

Since

$$
L_{k} \phi_{\lambda, k}=-\sum_{j=1}^{n}\left(c_{k j}-\delta_{k j}\left(\lambda+\mu_{\lambda}\right)\right) \phi_{\lambda, j},
$$

we see that $L_{k} \phi_{\lambda, k}$ remain bounded in $L^{\infty}(\Omega)$ as $\lambda \rightarrow \lambda_{1}$, for all $k=1, \ldots, n$. Classical interior elliptic estimates for scalar equations imply

$$
\left\|\Phi_{\lambda}\right\|_{W^{2, q}\left(\Omega^{\prime}\right)} \leqslant C,
$$

for any $1<q<\infty$ and any $\Omega^{\prime} \Subset \Omega$.

We infer from (149) that there exists a sequence $\left\{\lambda^{(j)}\right\}_{j=1}^{\infty}$ and a function $\Phi_{1}$ such that

$$
\lambda^{(j)} \rightarrow \lambda_{1} \quad \text { and } \quad \Phi^{(j)}=\Phi_{\lambda^{(j)}} \rightarrow \Phi_{1}
$$

weakly in $W_{\text {loc }}^{2, q}(\Omega), 1<q<\infty$, and uniformly in any compact subset of $\Omega$. It follows that $\Phi_{1}$ solves the equation

$$
\left(L+\mathcal{C}+\lambda_{1} I\right) \Phi_{1}=0 \quad \text { in } \Omega
$$

(note that (143) implies $\lambda^{(j)}+\mu_{\lambda^{(j)}} \rightarrow \lambda_{1}$ ), and

$$
0 \leqslant \Phi_{1} \leqslant C_{3} e \text { in } \Omega .
$$

Since, by (144), $\Phi_{1}\left(x_{0}\right) \geqslant e$, the strong maximum principle implies $\Phi_{1}>0$ in $\Omega$.

Finally,

$$
\begin{aligned}
L \Phi^{(j)} & =-\mathcal{C} \Phi^{(j)}-\left(\lambda^{(j)}+\mu_{\lambda^{(j)}}\right) \Phi^{(j)} \\
& \geqslant-v C_{3} e-\frac{C_{1} C_{3}}{R^{2}} e=-C_{4} e \quad \text { in } \Omega,
\end{aligned}
$$

so the usual maximum principle for scalar equations implies

$$
\Phi^{(j)} \leqslant C_{4} \Phi_{0} \quad \text { in } \Omega,
$$

where $\Phi_{0}$ is the solution of the problem

$$
\begin{cases}L \Phi_{0}=-e & \text { in } \Omega, \\ \Phi_{0}=0 & \text { on } \partial \Omega .\end{cases}
$$

Hence

$$
0<\Phi_{1} \leqslant C_{4} \Phi_{0} \quad \text { in } \Omega,
$$

so $\Phi_{1} \in C(\bar{\Omega})$ and $\Phi_{1}=0$ on $\partial \Omega$.

Statements (a) and (e) of Theorem 13.1 are proved.

The first part of statement (b) follows from the result of Sweers (Theorem 1.1 in [40]). Indeed, if $\bar{\lambda}<\lambda_{1}$ is an eigenvalue for $-(L+\mathcal{C})$ then the operator $L+\mathcal{C}+\frac{\lambda_{1}+\bar{\lambda}}{2} I$ satisfies the hypotheses of Theorem 1.1 in [40], and hence does not have negative eigenvalues - a contradiction. The second part of (b) is a particular case of (d).

Let us prove statements (c) and (d). It is clear that the assumptions in (c) imply $\lambda_{1} \geqslant 0$. If $\lambda_{1}=0$, set

$$
\tau=\sup \left\{t \in \mathbb{R} \mid \Psi \geqslant t \Phi_{1} \text { in } \Omega\right\} .
$$

By continuity $\Psi \geqslant \tau \Phi_{1}$. If $\Psi=\tau \Phi_{1}$, we are done. If not, the strong maximum principle, applied to

$$
(L+\mathcal{C})\left(\Psi-\tau \Phi_{1}\right) \leqslant 0,
$$


implies $\Psi>\tau \Phi_{1}$ in $\Omega$. We now make use of the maximum principle in "small domains" obtained by de Figueiredo in [14] (this result is stated in Corollary 14.1 below). It follows from this result that if a compact set $K \subset \Omega$ is such that meas $(\Omega \backslash K)$ is small enough, then $L+\mathcal{C}$ satisfies the maximum principle in $\Omega \backslash K$. Since $K$ is compact, there exists $\varepsilon>0$ such that $\Psi \geqslant(\tau+\varepsilon) \Phi_{1}$ in $K$. Since

$$
\begin{cases}(L+\mathcal{C})\left(\Psi-(\tau+\varepsilon) \Phi_{1}\right) \leqslant 0 & \text { in } \Omega \backslash K \\ \Psi-(\tau+\varepsilon) \Phi_{1} \geqslant 0 & \text { on } \partial(\Omega \backslash K)\end{cases}
$$

we get, by the maximum principle, $\Psi \geqslant(\tau+\varepsilon) \Phi_{1}$ in $\Omega$, which contradicts (150).

Finally, let us prove (d). We can suppose that $\lambda_{1}=0$ (replace $\mathcal{C}$ by $\mathcal{C}+\lambda_{1} I$ ). It suffices to find $\alpha>0$ such that

$$
Z=\bar{\Psi}+\alpha \Phi_{1}>0 \text { in } \Omega
$$

( $Z$ then satisfies the assumption in (c)). We fix a compact $K \subset \Omega$ such that $L+\mathcal{C}$ satisfies the maximum principle in $\Omega \backslash K$ and we take $\alpha>0$ such that $Z>0$ in $K$. Since

$$
\begin{cases}(L+\mathcal{C}) Z \leqslant 0 & \text { in } \Omega \backslash K \\ Z \geqslant 0 & \text { on } \partial(\Omega \backslash K)\end{cases}
$$

we get $Z>0$ in $\Omega \backslash K$.

Theorem 13.1 is proved.

At the end of this section we recall the following result of Sweers [40].

Theorem 13.2 (Sweers). Suppose $\mathcal{C}$ is irreducible and let $\vec{f} \in L^{q}\left(\Omega, \mathbb{R}^{n}\right), q \geqslant N$. If $\lambda_{1}>0$ then there exists a unique solution in $W_{\text {loc }}^{2, q}\left(\Omega, \mathbb{R}^{n}\right) \cap C\left(\bar{\Omega}, \mathbb{R}^{n}\right)$ of the problem

$$
\begin{cases}L u+\mathcal{C} u=-\vec{f} & \text { in } \Omega, \\ u=0 & \text { on } \partial \Omega\end{cases}
$$

Moreover, $\vec{f} \geqslant 0$ in $\Omega$ implies $u \geqslant 0$ in $\Omega ; \vec{f}=\left(f_{1}, \ldots, f_{n}\right) \geqslant 0$ and $f_{i} \not \equiv 0$, for some $i$, imply $\vec{f}>0$.

\section{Necessary and sufficient conditions for a linear system to satisfy the maximum principle}

The existence of a principal eigenfunction permits us to show that condition $(\Psi)$ in Theorem 8.1(a), which has long been known to be sufficient for the maximum principle, is also necessary for its validity. Our result, applied to a fully coupled system, says the maximum principle holds if and only if the principal eigenvalue of the matrix operator $L+\mathcal{C}$ is positive. This result contains the well-known necessary and sufficient condition for a scalar operator to verify the maximum principle.

Let the matrix $\mathcal{C}$ be written in the form

$$
\mathcal{C}=\left(\mathcal{C}_{k l}\right)_{k, l=1}^{m}
$$

in $\Omega$, where, we recall, $1 \leqslant m \leqslant n, \mathcal{C}_{k l}$ are $t_{k} \times t_{l}$ matrices, $\sum_{k=1}^{m} t_{k}=n, \mathcal{C}_{k k}$ is an irreducible matrix for all $k=1, \ldots, m$, and $\mathcal{C}_{k l} \equiv 0$ for all $k, l \in\{1, \ldots, m\}$ with $k<l$. We have set $s_{0}=0, s_{k}=\sum_{i=1}^{k} t_{i}$, and $S_{k}=\left\{s_{k-1}+1, \ldots, s_{k}\right\}$. 
Let $\lambda_{1}^{(k)} \in \mathbb{R}$ and $\Phi_{1}^{(k)} \in W_{\text {loc }}^{2, q}\left(\Omega, \mathbb{R}^{t_{k}}\right) \cap C\left(\bar{\Omega}, \mathbb{R}^{t_{k}}\right), \forall q<\infty$, be the principal eigenvalue and eigenfunction of the operator $L^{k}+\mathcal{C}_{k k}$ in $\Omega$, where we denote $L^{k}=\operatorname{diag}\left(L_{s_{k-1}+1}, \ldots, L_{s_{k}}\right)$. The existence of $\lambda_{1}^{(k)}$ and $\Phi_{1}^{(k)}$ is ensured by Theorem 13.1.

Theorem 14.1. Let (L1) through (L3) hold and let $a_{i j}^{k}$ be continuous functions, for all $i, j, k$. The following are equivalent:

(i) $L+\mathcal{C}$ satisfies the maximum principle in $\Omega$, in the sense described in the beginning of Section 11;

(ii) $\lambda_{1}^{(k)}>0$, for all $k \in\{1, \ldots, m\}$;

(iii) there exists a function $\Psi>0$ in $\Omega$ such that $(L+\mathcal{C}) \Psi \leqslant 0$ in $\Omega$, and either $\Psi \not \equiv 0$ on $\partial \Omega$ or $(L+\mathcal{C}) \Psi \not \equiv 0$ in $\Omega$ (component-wise).

Proof. (i) $\Rightarrow$ (ii). Suppose there exists $k \in\{1, \ldots, m\}$ such that $\lambda_{1}^{(k)} \leqslant 0$. Set

$$
\Psi=\left(0, \ldots, 0, \Phi_{1}^{(k)}, 0, \ldots, 0\right)
$$

(the only non-zero coordinates of $\Psi$ are those with indices in $S_{k}$ ). Then

$$
(L+\mathcal{C}) \Psi=\left(0, \ldots, 0,-\lambda_{1}^{(k)} \Phi_{1}^{(k)}, \mathcal{C}_{k+1 k} \Phi_{1}^{(k)}, \ldots, \mathcal{C}_{m k} \Phi_{1}^{(k)}\right),
$$

so, by (L3),

$$
\begin{cases}(L+\mathcal{C}) \Psi \geqslant 0 & \text { in } \Omega \\ \Psi=0 & \text { on } \partial \Omega\end{cases}
$$

but $\Psi \nless 0$ in $\Omega$, which contradicts the maximum principle.

(ii) $\Rightarrow$ (iii). We use a recurrent procedure to construct $\Psi=\left(\Psi^{(1)}, \ldots, \Psi^{(m)}\right)\left(\Psi^{(k)}\right.$ consists of $t_{k}$ components). Set $\Psi^{(1)}=\Phi_{1}^{(1)}$. If $\mathcal{C}_{21} \equiv 0$ we take $\Psi^{(2)}=\Phi_{1}^{(2)}$. If $\mathcal{C}_{21} \not \equiv 0$ we take $\Psi^{(2)}$ to be the solution of the problem

$$
\begin{cases}\left(L^{2}+\mathcal{C}_{22}+\frac{\lambda_{1}^{(2)}}{2}\right) \Psi^{(2)}=-\mathcal{C}_{21} \Psi^{(1)} & \text { in } \Omega, \\ \Psi^{(2)}>0 & \text { in } \Omega, \\ \Psi^{(2)}=0 & \text { on } \partial \Omega .\end{cases}
$$

This boundary value problem is solvable, by Theorem 13.2.

Finally, for any $l \in\{2, \ldots, n\}$, when we have constructed $\Psi^{(1)}, \ldots, \Psi^{(l-1)}$, we take $\Psi^{(l)}$ to be either $\Phi_{1}^{(l)}$, in case $\mathcal{C}_{l k} \equiv 0$ for all $k=1, \ldots, l-1$, or the positive solution of the problem

$$
\begin{cases}\left(L^{l}+\mathcal{C}_{l l}+\frac{\lambda_{1}^{(l)}}{2}\right) \Psi^{(l)}=-\sum_{k=1}^{l-1} \mathcal{C}_{l k} \Psi^{(k)} & \text { in } \Omega, \\ \Psi^{(l)}=0 & \text { on } \partial \Omega,\end{cases}
$$

in case the right-hand side in (153) is not identically zero.

Then we have $\Psi>0$ and

$$
(L+\mathcal{C}) \Psi \leqslant\left(-\lambda_{1}^{(1)} \Phi_{1}^{(1)},-\frac{\lambda_{1}^{(2)}}{2} \Phi_{1}^{(2)}, \ldots,-\frac{\lambda_{1}^{(m)}}{2} \Phi_{1}^{(m)}\right)<0 \quad \text { in } \Omega .
$$

(iii) $\Rightarrow$ (ii). Let us note $\Psi=\left(\Psi^{(1)}, \ldots, \Psi^{(m)}\right)>0$. Since (L3) holds, we have

$$
\left(L^{k}+\mathcal{C}_{k k}\right) \Psi^{(k)} \leqslant 0
$$


and either $\Psi^{(k)} \not \equiv 0$ on $\partial \Omega$ or $\left(L^{k}+\mathcal{C}_{k k}\right) \Psi^{(k)} \not \equiv 0$ in $\Omega$, for all $k=1, \ldots, m$. It follows from Theorem 13.1(c) that either $\lambda_{1}^{(k)}>0$ or $\lambda_{1}^{(k)}=0$ and, in the latter case, $\Psi^{(k)}=$ const $\cdot \Phi_{1}^{(k)}$. The last equality is impossible, by (iii) and the properties of $\Phi_{1}$.

(iii) $\Rightarrow$ (i). We show that (iii) is equivalent to condition $(\Psi)$ in the ABP estimate (Theorem 8.1(a)). Then the conclusion is immediate, since the maximum principle is a particular case of the ABP estimate.

Lemma 14.1. Suppose $\lambda_{1}^{(k)}>0$, for all $k=1, \ldots, m$. Then there exists a function $\bar{\Psi} \in W_{\mathrm{loc}}^{2, q}\left(\Omega, \mathbb{R}^{n}\right) \cap$ $C\left(\bar{\Omega}, \mathbb{R}^{n}\right), \forall q<\infty$, such that

$$
\begin{cases}(L+\mathcal{C}) \bar{\Psi} \leqslant 0 & \text { in } \Omega \\ \bar{\Psi} \geqslant e & \text { in } \Omega .\end{cases}
$$

Proof. Since (iii) is equivalent to (ii) we can consider the vector $\Psi$ constructed in the proof of (ii) $\Rightarrow$ (iii). Then we can adapt to our situation the proof of Lemma 6.1 in [2]. Let us sketch the argument.

We take a compact set $K \subset \Omega$ and solve the following $n$ scalar equations

$$
\begin{cases}L W=-2 v e & \text { in } \Omega \backslash K, \\ L W=0 & \text { in } K, \\ W=0 & \text { on } \partial \Omega .\end{cases}
$$

As in [2], by taking $K$ sufficiently close to $\Omega$, we can ensure that $W<e$ in $\Omega$. Taking $\varepsilon_{0}$ such that $\Psi \geqslant \varepsilon_{0} e$ on $K$ and setting

$$
a=\max _{1 \leqslant k \leqslant m} \frac{4 v}{\lambda_{1}^{(k)} \varepsilon_{0}} \text { and } \bar{\Psi}=e+W+a \Psi,
$$

we get $(L+\mathcal{C}) \bar{\Psi} \leqslant 0$ in $\Omega$, as in [2].

Theorem 14.1 is proved.

Finally, we give a lower bound for the principal eigenvalue, analogous to the estimate in Lemma 4.1 in [2].

Proposition 14.1. Under the conditions of Theorem 13.1, set

$$
b_{1}=\max _{1 \leqslant j \leqslant n}\left\{\sum_{i \neq j}\left\|c_{i j}\right\|_{L^{\infty}(\Omega)}+\left\|c_{j j}^{+}\right\|_{L^{\infty}(\Omega)}\right\} .
$$

Then

$$
\lambda_{1} \geqslant \frac{1}{C_{\mathrm{ABP}}|\Omega|^{1 / N}}-b_{1} .
$$

Proof. Apply the ABP estimate to

$$
\left(L+\mathcal{C}-b_{1} I\right) \Phi_{1}=-\left(b_{1}+\lambda_{1}\right) \Phi_{1} .
$$

The following maximum principle in small domains (see [14]) is a consequence of Proposition 14.1 and Theorem 14.1.

Corollary 14.1. Suppose (L1)-(L3) hold. For any $d>0$ there exists a positive number $\delta=\delta\left(N, c_{0}, v, d\right)$ such that $\operatorname{diam} \Omega<d$ and $|\Omega|<\delta$

imply that the operator $L+\mathcal{C}$ satisfies the maximum principle in $\Omega$. 


\section{Higher order equations. Estimates for equations of polyharmonic type}

The equation $\Delta^{n} u=0, n \geqslant 2$, is very classical. It is known that solutions of this equation (called polyharmonic functions) do not admit the same estimates as harmonic functions. However, there is an important subclass of polyharmonic functions (the so-called completely superharmonic functions, see below) for which Harnack and Liouville type results have been obtained. The possibility of extending these results to equations with right-hand side and zero-order term has been an open question for a long time. Our estimates for systems permit us to give an affirmative answer to this question.

Our results give ABP and Harnack estimates for equations of arbitrary order in the form

$$
\left(-L_{n}-c_{n}(x)\right) \cdots\left(-L_{1}-c_{1}(x)\right) u-c(x) u=f(x) \text { in } \Omega
$$

(in contrast to the rest of the paper, in this section $u$ and $f$ denote scalar functions). It is easy to see that (154) is equivalent to the system

$$
L U+\mathcal{C} U=\vec{f},
$$

where

$$
\mathcal{C}(x)=\left(\begin{array}{ccccc}
c_{1}(x) & 1 & 0 & \ldots & 0 \\
0 & c_{2}(x) & 1 & \ldots & 0 \\
\ldots & \ldots & \ldots & \ddots & \ldots \\
0 & 0 & 0 & \ldots & 1 \\
c(x) & 0 & 0 & \ldots & c_{n}(x)
\end{array}\right), \quad U=\left(\begin{array}{c}
u \\
\left(-L_{1}-c_{1}\right) u \\
\vdots \\
\left(\prod_{i=1}^{n-1}\left(-L_{i}-c_{i}\right)\right) u
\end{array}\right)
$$

and $\vec{f}=(0, \ldots, 0, f)^{\mathrm{T}}$. Note that the matrix $\mathcal{C}$ is fully coupled when $c \not \equiv 0$ and is in triangular form when $c \equiv 0$.

The simplest and most studied example for (154) is the biharmonic equation

$$
-\Delta(\Delta u)+c(x) u=f(x) \text { in } \Omega,
$$

which corresponds to the system

$$
\left\{\begin{array}{l}
\Delta u+v=0 \\
\Delta v+c(x) u=f(x)
\end{array}\right.
$$

We have the following Harnack estimate for Eq. (154). We give separately the Harnack estimate for the polyharmonic equation with a right-hand side.

Theorem 15.1. Let $f \in L^{N}(\Omega)$.

(a) Suppose that $u \in C^{2 n-2}(\Omega)$ satisfies the equation

$$
(-\Delta)^{n} u=f
$$

in the viscosity sense in $\Omega$ and

$$
(-\Delta)^{k} u \geqslant 0
$$

in a ball $B_{3 R} \subset \Omega$, for $k=0, \ldots, n-1$.

Then

$$
\sup _{B_{R}} u \leqslant C\left(\inf _{B_{R}} u+R\|f\|_{L^{N}\left(B_{3 R}\right)}\right),
$$

where $C=C(n, N, R)$.

(b) Let $c \in L^{\infty}$ and $c_{i} \in L^{\infty}(\Omega), i=1, \ldots, n$, be functions such that

$$
c \not \equiv 0, \quad 0 \leqslant c \leqslant v, \quad \max _{1 \leqslant k \leqslant n}\left\|c_{k}\right\|_{L^{\infty}(\Omega)} \leqslant v .
$$


Let $u \in C^{2 n-2}(\Omega)$ be a non-negative viscosity solution of

$$
\left(-L_{n}-c_{n}\right) \cdots\left(-L_{1}-c_{1}\right) u-c u=f \quad \text { in } \Omega
$$

such that

$$
\left(-L_{k}-c_{k}\right) \cdots\left(-L_{1}-c_{1}\right) u \geqslant 0
$$

for all $k=1, \ldots, n-1$.

Then

$$
\sup _{B_{R}}\left\{u \vee \max _{1 \leqslant k \leqslant n-1}\left(\prod_{i=1}^{k}\left(-L_{i}-c_{i}\right)\right) u\right\} \leqslant C\left(\inf _{B_{R}}\left\{u \wedge \min _{1 \leqslant k \leqslant n-1}\left(\prod_{i=1}^{k}\left(-L_{i}-c_{i}\right)\right) u\right\}+R\|f\|_{L^{N}\left(B_{3 R}\right)}\right) .
$$

Functions which satisfy condition (159) are called completely superharmonic (of order $n-1$ ). It is easy to see that this hypothesis cannot be omitted in Theorem 15.1 (take for example $u=|x|^{2}$ in the unit ball; then the weak Harnack inequality fails, since $\Delta^{2} u=0, u \geqslant 0, u(0)=0$, but $\left.u \neq \equiv 0\right)$.

The particular role of completely superharmonic functions was already noticed by M. Nicolesco in his classical book [36, pp. 16-25]. He proved that the coefficients in the Almansi expansion of a polyharmonic function which satisfies this property are of constant sign, as well as a Harnack convergence type theorem for such functions. Harnack type results for positive solutions of $\Delta^{n} u=0$ were obtained by many authors, mostly by studying spherical means of $u$ (see, for instance, [18,20]). An interesting Liouville type result for completely superharmonic functions was proved by [37]; other theorems of Liouville type can be found in [19]. The inequality (160) for $f \equiv 0$ and $n=2$ appears for example in [39] (we could not find a reference for $n>2$ ). All these results rely heavily on the polyharmonicity of the function and could not be extended to equations with a non-trivial right-hand side. Quite little is known about the equation $(-\Delta)^{n} u-c(x) u=0$ either (see also Theorem 15.2).

Recently, using Green functions, Grunau and Sweers (see [23, Theorem 5.1]) obtained maximum principle type results for classical solutions of (154), in the case when the domain is a ball and all derivatives of $u$ of order smaller than $n$ vanish on $\partial \Omega$. In [24] they used this result to obtain a local maximum principle for equations of order $2 n$, provided the lower order coefficients are sufficiently small.

Theorem 13.1 permits us to define a "principal eigenvalue" and a "principal eigenfunction" for the operator $(-\Delta)^{n}-c(x), c \geqslant 0$, under Dirichlet boundary conditions for the lower order Laplacians. The positivity of this eigenvalue is a necessary and sufficient condition for the operator to satisfy the maximum principle. Note that the existence of a first eigenvalue for the other classical polyharmonic boundary value problem (Lauricella's problem) - in which the boundary conditions require that $D^{k} u=0$ on $\partial \Omega$ for $k=0, \ldots, n-1-$ is wellknown.

\section{Theorem 15.2.}

(a) Let $c \in L^{\infty}(\Omega), 0 \leqslant c \leqslant v$. There exists a real number $\lambda_{1}=\lambda_{1}^{(c)}$ and a function $\phi_{1}=\phi_{1}^{(c)}$ in $W_{\mathrm{loc}}^{2 n, q}(\Omega) \cap$ $C^{2 n-2}(\bar{\Omega}), \forall q<\infty$, such that

$$
\begin{aligned}
& \left(-\Delta-\lambda_{1}\right)^{n} \phi_{1}-c \phi_{1}=0 \quad \text { in } \Omega, \\
& \phi_{1}>0, \quad\left(-\Delta-\lambda_{1}\right)^{k} \phi_{1}>0 \quad \text { in } \Omega, \\
& \phi_{1}=0, \quad\left(-\Delta-\lambda_{1}\right)^{k} \phi_{1}=0 \quad \text { on } \partial \Omega,
\end{aligned}
$$

for $k=1, \ldots, n-1$. There are no eigenvalues (with the Dirichlet condition (164)) smaller than $\lambda_{1}$ and $\left(\lambda_{1}, \phi_{1}\right)$ is the unique couple (up to a normalization of $\phi_{1}$ ), which satisfies (162), (163) and (164). 
(b) $\lambda_{1}^{(c)}>0$ is a necessary and sufficient condition for the operator $(-\Delta)^{n}-c$ to satisfy the maximum principle, in the following sense

$$
\begin{cases}(-\Delta)^{n} u-c u \leqslant 0 & \text { in } \Omega \\ (-\Delta)^{k} u \leqslant 0 & \text { on } \partial \Omega, k=0, \ldots, n-1,\end{cases}
$$

implies $(-\Delta)^{k} u \leqslant 0$ in $\Omega$, for $k=0, \ldots, n-1$.

(c) Let $\phi_{1}^{(c)}$ be normalized so that $\phi_{1}^{(c)}\left(x_{0}\right)=1$, for some $x_{0} \in \Omega$. Then

$$
\sup _{\Omega} \phi_{1}^{(c)} \leqslant C(n, N, v, \Omega) .
$$

(d) If $\left\{c^{j}\right\}_{j=1}^{\infty}$ is a sequence such that $c^{j} \geqslant 0,\left\{c^{j}\right\}$ is bounded in $L^{\infty}(\Omega)$ and $\left\{c^{j}\right\}$ tends to zero a.e. in $\Omega$, then $\lambda_{1}^{\left(c_{j}\right)}$ tends to the principal eigenvalue of the Laplacian in $\Omega$ and

$$
\begin{aligned}
\phi_{1}^{\left(c_{j}\right)} & \rightarrow \phi_{1}(\Delta), \\
\left(-\Delta-\lambda_{1}^{\left(c_{j}\right)}\right)^{k} \phi_{1}^{\left(c_{j}\right)} & \rightarrow 0, \quad k=1, \ldots, n-1,
\end{aligned}
$$

weakly in $W_{\mathrm{loc}}^{2, q}(\Omega)$ and strongly in $C(\bar{\Omega})$ (the functions $\phi_{1}^{\left(c_{j}\right)}$ are assumed to be normalized as in $(\mathrm{c})$ ).

Proof. In view of Theorem 13.1 and the representation (155), only part (d) needs a proof. Suppose for simplicity $n=2$ (the case $n>2$ is very much the same).

Set $\lambda^{j}=\lambda_{1}^{\left(c_{j}\right)}$ and $\phi^{j}=\phi_{1}^{\left(c_{j}\right)}$. It follows from (143) and Proposition 14.1 that the sequence $\left\{\lambda^{j}\right\}$ is bounded. Hence (up to a subsequence) it converges to a number $\bar{\lambda}$.

We have

$$
\left\{\begin{array}{l}
\left(-\Delta-\lambda^{j}\right) u^{j}=v^{j} \\
\left(-\Delta-\lambda^{j}\right) v^{j}=c^{j} u^{j},
\end{array}\right.
$$

where $u^{j}=\phi^{j}$ and $v^{j}=\left(-\Delta-\lambda^{j}\right) \phi^{j}$.

Applying the theorem of Krylov we already used in Section 7 (Theorem 7.1 on page 33), we obtain from the first equation in (165)

$$
\inf _{K} u^{j} \geqslant C(N, v, K, \Omega) \inf _{K} v^{j},
$$

for any compact subset $K \subset \Omega$.

Applied to (165), our local maximum principle (Proposition 8.1) yields

$$
\sup _{B_{R}} u^{j} \vee v^{j} \leqslant C\left(\left|u^{j}\right|_{p, 2 R}+\left|v^{j}\right|_{p, 2 R}\right),
$$

for any $p>0$, any $B_{2 R} \subset \Omega$, with $C=C\left(p, N, v R^{2}\right)$. The weak Harnack inequality for scalar equations (Theorem 9.22 in [22]) applied to

$$
\left(-\Delta-\lambda^{j}\right) u^{j} \geqslant 0 \text { and }\left(-\Delta-\lambda^{j}\right) v^{j} \geqslant 0
$$

then gives

$$
\begin{aligned}
\sup _{B_{R}} u^{j} \vee v^{j} & \leqslant C\left(\inf _{B_{R}} u^{j}+\inf _{B_{R}} v^{j}\right) \\
& \leqslant C \inf _{B_{R}} u^{j}=C(N, v, \Omega)
\end{aligned}
$$


if $x_{0} \in B_{R}$, where we have used (166), (167), and $u^{j}\left(x_{0}\right)=1$. Hence, for any compact set $K \subset \Omega$,

$$
\sup _{K} u^{j} \vee v^{j} \leqslant C(N, v, K, \Omega) .
$$

By proceeding as in the proof of Theorem 13.1, that is, by taking $K$ close enough to $\Omega$ and by applying the ABP inequality in $\Omega \backslash K$, we get

$$
\left\|u^{j} \vee v^{j}\right\|_{L^{\infty}(\Omega)} \leqslant C(N, v, \Omega)
$$

Then elliptic theory implies

$$
u^{j} \rightarrow u \text { and } \quad v^{j} \rightarrow v
$$

weakly in $W_{\text {loc }}^{2, q}(\Omega), \forall q<\infty$, and strongly in $C(\bar{\Omega})$. By passing to the limit in (165) we obtain

$$
\begin{cases}(-\Delta-\bar{\lambda}) u=v & \text { in } \Omega, \\ (-\Delta-\bar{\lambda}) v=0 & \text { in } \Omega, \\ u=v=0 & \text { on } \partial \Omega .\end{cases}
$$

Since $v \geqslant 0$, we infer from the second equation in (168) that either $v \equiv 0$ or $\bar{\lambda}=\lambda_{1}(\Delta)$ and $v=\phi_{1}(\Delta)>0$. In the second case we obtain a contradiction with the first equation, since

$$
\begin{cases}\left(-\Delta-\lambda_{1}(\Delta)\right) u>0 & \text { in } \Omega \\ u=0 & \text { on } \partial \Omega\end{cases}
$$

has no solution. Hence

$$
\begin{cases}(-\Delta-\bar{\lambda}) u=0 & \text { in } \Omega \\ u=0 & \text { on } \partial \Omega\end{cases}
$$

Since $u \geqslant 0$ and $u\left(x_{0}\right)=1$, we get $\bar{\lambda}=\lambda_{1}(\Delta)$ and $u=\phi_{1}(\Delta)$.

Theorem 15.2 is proved.

More generally, for any $n$ elliptic operators $L_{1}, \ldots, L_{n}$, satisfying (H1)-(H2), and any $c \geqslant 0$, there exists a couple $\left(\lambda_{1}, \phi_{1}\right)$ such that

$$
\left(-L_{n}-\lambda_{1}\right) \cdots\left(-L_{1}-\lambda_{1}\right) \phi_{1}-c \phi_{1}=0,
$$

with the appropriate positivity and boundary conditions.

\section{References}

[1] A. Arapostathis, M. Ghosh, St. Marcus, Harnack's inequality for cooperative weakly coupled elliptic systems, Comm. Partial Differential Equations 24 (9-10) (1999) 1555-1571.

[2] H. Berestycki, L. Nirenberg, S.R.S. Varadhan, The principal eigenvalue and maximum principle for second order elliptic operators in general domains, Comm. Pure Appl. Math. 47 (1) (1994) 47-92.

[3] I. Birindelli, E. Mitidieri, G. Sweers, Existence of the principal eigenfunction for cooperative elliptic systems in a general domain, Differential Equations (Differentsial'nye Uravneniya) 35 (3) (1999) (in Russian).

[4] M. Bladt, A Markov modulated financial model, J. Comm. Statist.: Stoch. Models 14 (1998) 225-240.

[5] M. Bladt, P. Padilla, Nonlinear financial models: finite Markov modulation and limits, Preprint.

[6] J. Busca, Existence results for Bellman equations and maximum principles in unbounded domains, Comm. Partial Differential Equations 24 (11-12) (1999) 2023-2042.

[7] L.A. Caffarelli, Interior estimates for fully nonlinear elliptic equations, Ann. of Math. 130 (1989) 189-213.

[8] L.A. Caffarelli, X. Cabre, Fully Nonlinear Elliptic Equations, in: Amer. Math. Soc. Collog. Publ., vol. 43, American Mathematical Society, Providence, RI, 1995. 
[9] L.A. Caffarelli, M.G. Crandall, M. Kocan, A. Świech, On viscosity solutions of fully nonlinear equations with measurable ingredients, Comm. Pure Appl. Math 49 (1996) 365-397.

[10] Z.Q. Chen, Z. Zhao, Harnack principle for weakly coupled elliptic systems, J. Differential Equations 39 (1997) 261-282.

[11] Z.Q. Chen, Z. Zhao, Potential theory for elliptic systems, Ann. Probab. 24 (1) (1996) 293-319.

[12] M.G. Crandall, H. Ishii, P.-L. Lions, User's guide to viscosity solutions of second-order partial differential equations, Bull. Amer. Math. Soc. 27 (1) (1992) 1-67.

[13] M.G. Crandall, M. Kocan, A. Świech, $L^{p}$ theory for fully nonlinear uniformly parabolic equations, Comm. Partial Differential Equations 25 (11\&12) (2000) 1997-2053.

[14] D.G. de Figueiredo, Monotonicity and symmetry of solutions of elliptic systems in general domains, Nonlinear Differential Equations Appl. 1 (1994) 119-123.

[15] D.G. de Figueiredo, E. Mitidieri, Maximum principles for linear elliptic systems, Rend. Inst. Mat. Univ. Trieste (1992) 36-66.

[16] D.G. de Figueiredo, E. Mitidieri, Maximum principles for cooperative elliptic systems, C. R. Acad. Sci. Paris, Ser. I 310 (2) (1990) $49-52$.

[17] E. de Giorgi, Un esempio di estremali discontinue per un problema variazionale di tipo elliptico, Boll. Un. Mat. Ital. 1 (4) (1968) $135-137$.

[18] R.J. Duffin, Z. Nehari, Note on polyharmonic functions, Proc. Amer. Math. Soc. 12 (1961) 110-115.

[19] A. Friedman, On $n$-metaharmonic functions and harmonic functions of infinite order, Proc. Amer. Math. Soc. 8 (1957) $223-229$.

[20] M. Ghermanescu, Sur les valeurs moyennes des fonctions, Math. Ann. 119 (1944) 288-320.

[21] M. Giaquinta, Multiple Integrals in the Calculus of Variations and Nonlinear Elliptic Systems, in: Ann. Math. Stud., vol. 105, Princeton University Press, 1983.

[22] D. Gilbarg, N.S. Trudinger, Elliptic Partial Differential Equations of Second Order, 2nd edition, Revised Third Printing, Springer-Verlag, 1998.

[23] H. Grunau, G. Sweers, Positivity for equations involving polyharmonic operators with Dirichlet boundary conditions, Math. Ann. 307 (1997) 589.

[24] H. Grunau, G. Sweers, Classical solutions for some higher order semilinear elliptic equations under weak growth conditions, Nonlinear Anal. 28 (1997) 799-807.

[25] P. Hess, On the eigenvalue problem for weakly coupled elliptic systems, Arch. Rational Mech. Anal. 81 (2) (1983) 151-159.

[26] S. Hildebrandt, K.-O. Widman, On the Hölder continuity of weak solutions of quasilinear elliptic systems of second order, Ann. Sc. Norm. Sup. Pisa Cl. Sci. 4 (1) (1977) 145-178.

[27] H. Ishii, S. Koike, Viscosity solutions for monotone systems of second-order elliptic PDE's, Comm. Partial Differential Equations 16 (6 \& 7) (1991) 1095-1128.

[28] R. Jensen, The maximum principle for viscosity solutions of fully nonlinear seoncd order partial differential equations, Arch. Rational Mech. Anal. 101 (1988) 1-27.

[29] R. Jensen, P.L. Lions, P.E. Souganidis, A uniqueness result for viscosity solutions of fully nonlinear second order partial differential equations, Proc. Amer. Math. Soc. 4 (1988) 975-978.

[30] Krylov, Nonlinear Elliptic and Parabolic Equations of Second Order, in: Coll. Math. Appl., 1987.

[31] J.-M. Lasry, P.-L. Lions, Large deviations for diffusion process coupled by a jump process, C. R. Acad. Sci. Paris, Sér. I 321 (1995) 849-854.

[32] S.M. Lenhart, S.A. Belbas, A system of nonlinear PDE's arising in the optimal control of stochastic systems, SIAM J. Appl. Math. 43 (1983) 465-475.

[33] Mandras, Diseguanza di Harnack per sistemi elliptici debolmente accopiati, Boll. Un. Mat. Ital. A 14 (5) (1977) 313-332.

[34] J.D. Murray, Mathematical Biology, Springer-Verlag, 1993.

[35] C. Muscalu, On the Harnack principle for strongly elliptic systems with nonsmooth coefficients, Comm. Pure Appl. Math. 52 (1999) $1213-1230$.

[36] M. Nicolescu, Les fonctions polyharmoniques, Hermann \& Cie, Paris, 1936.

[37] I.E. Ovčarenko, On multiply superharmonic functions, Uspekhi Math. Nauk 16 (3) (1961) 197-200 (in Russian).

[38] J. Serrin, Local behaviour of solutions of quasilinear equations, Acta Math. 111 (1964) 247-302.

[39] E.P. Smyrnelis, Une propriété de moyenne des fonctions biharmoniques, Bull. Sci. Math. (2) 109 (1985) 103-111.

[40] G. Sweers, Strong positivity in $C(\bar{\Omega})$ for elliptic systems, Math. Z. 209 (1992) 251-271.

[41] L. Wang, On the regularity theory of fully nonlinear parabolic equations: I, Comm. Pure Appl. Math. 45 (1) (1992) $27-76$. 